\title{
Preserving Global Exponential Stability of Hybrid BAM Neural Networks with Reaction Diffusion Terms in the Presence of Stochastic Noise and Connection Weight Matrices Uncertainty
}

\author{
Yan $\mathrm{Li}^{1,2}$ and Yi Shen ${ }^{1}$ \\ ${ }^{1}$ Department of Control Science and Engineering, Huazhong University of Science and Technology, Wuhan 430074, China \\ ${ }^{2}$ College of Science, Huazhong Agriculture University, Wuhan 430079, China \\ Correspondence should be addressed to Yi Shen; yeeshen0912@gmail.com
}

Received 8 February 2014; Accepted 6 March 2014; Published 17 April 2014

Academic Editor: Weihai Zhang

Copyright (C) 2014 Y. Li and Y. Shen. This is an open access article distributed under the Creative Commons Attribution License, which permits unrestricted use, distribution, and reproduction in any medium, provided the original work is properly cited.

\begin{abstract}
We study the impact of stochastic noise and connection weight matrices uncertainty on global exponential stability of hybrid BAM neural networks with reaction diffusion terms. Given globally exponentially stable hybrid BAM neural networks with reaction diffusion terms, the question to be addressed here is how much stochastic noise and connection weights matrices uncertainty the neural networks can tolerate while maintaining global exponential stability. The upper threshold of stochastic noise and connection weights matrices uncertainty is defined by using the transcendental equations. We find that the perturbed hybrid BAM neural networks with reaction diffusion terms preserve global exponential stability if the intensity of both stochastic noise and connection weights matrices uncertainty is smaller than the defined upper threshold. A numerical example is also provided to illustrate the theoretical conclusion.
\end{abstract}

\section{Introduction}

The bidirectional associative memory (BAM) neural networks were first introduced by Kosko in which the neurons in one layer are fully interconnected to the neurons in the other layer, while there are no interconnection among the neurons in the same layers [1-3]. The BAM neural networks widely have applications in pattern recognition, robot, signal processing, associative memory, solving optimization problems, and automatic control engineering. For most successful applications of BAM neural networks, the stability analysis on BAM neural networks is usually a prerequisite. The exponential stability and periodic oscillatory solution of BAM neural networks with delays were studied by Cao et al. $[4,5]$. Moreover, in BAM neural networks, diffusion phenomena can hardly be avoided when electrons are moving in asymmetric electromagnetic fields. The BAM neural networks with reaction diffusion terms described by partial differential equations were investigated by many authors [611]. Sometimes, it is necessary to assess the parameters of the neural network that may experience abrupt changes caused by certain phenomena such as component failure or repair, change of subsystem interconnection, and environmental disturbance. The continuous-time Markov chains have been used to model these parameter jumps [12-14]. These neural networks with Markov chains are usually called hybrid neural networks. The almost surely exponential stability, moment exponential stability, and stabilization of hybrid neural networks were also researched; see, for example, [1517]. By making use of impulsive control, Zhu and Cao [18] considered the stability of hybrid neural networks with mixed delay.

For neural networks with stochastic noise, the system is usually described by stochastic differential equations. The stability of stochastic neural networks with delay or reaction diffusion terms was extensively analyzed by using the Itô formula and the linear matrix inequality (LMI) methods [1822]. As is well known, stochastic noise is often the sources of instability and may destabilize the stable neural networks [23]. For stable hybrid BAM neural networks with reaction 
diffusion terms, it is interesting to determine how much noise the stochastic neural networks can tolerate while maintaining global exponential stability.

Moreover, the connection weights of neurons depend on certain resistance and capacitance values which include uncertainty. The robust stability about parameter matrices uncertainty in neural networks was investigated by many authors $[24,25]$. If the uncertainty in connection weights matrices is too large, the neural networks may be unstable. Therefore, for stable hybrid BAM neural networks with reaction diffusion terms, it is also interesting to determine how much connection weights matrices uncertainty the neural networks can also tolerate while maintaining global exponential stability.

In this paper, we will study the impact of stochastic noise and connection weight matrices uncertainty of hybrid BAM neural networks with reaction diffusion terms. We give the upper threshold of stochastic noise and connection weights matrices uncertainty defined by using the transcendental equations. We find that the perturbed hybrid BAM neural networks with reaction diffusion terms preserve global exponential stability if the intensity of both stochastic noise and connection weights matrices uncertainty is smaller than the defined upper threshold.

The remainder of this paper is organized as follows. Some preliminaries are given in Section 2. Section 3 discusses the impact of the stochastic noise on global exponential stability of these neural networks. Section 4 discusses the impact of the connection weight matrices uncertainty and stochastic noise on global exponential stability of these neural networks. Finally, an example with numerical simulation is given to illustrate the effectiveness of the obtained results in Section 5.

\section{Preliminaries}

Throughout this paper, unless otherwise specified, let $\left(\Omega, \mathscr{F},\left\{\mathscr{F}_{t}\right\}_{t \geq 0}, \mathbb{P}\right)$ be complete probability space with a filtration $\left\{\mathscr{F}_{t}\right\}_{t \geq 0}$ satisfying the usual conditions (i.e., it is increasing and right continuous while $\mathscr{F}_{0}$ contains all $\mathbb{P}$ null sets). Let $W(t)$ be a scalar Brownian motion (Wiener process) defined on the probability space. Let $A^{T}$ denote the transpose of $A$. If $A$ is a matrix, its operator norm is denoted by $\|A\|=\sup \{|A x|:|x|=1\}$, where $|\cdot|$ is the Euclidean norm. Let $r(t), t \geq 0$, be a right-continuous Markov chain on the probability space taking values in a finite state space $\mathbb{S}=\{1,2, \ldots, N\}$ with the generator $\Gamma=\left(\gamma_{p q}\right)_{N \times N}$ given by

$$
\begin{aligned}
& \mathbb{P}\{r(t+\Delta)=q \mid r(t)=p\} \\
& \quad= \begin{cases}\gamma_{p q} \Delta+o(\Delta) & \text { if } p \neq q \\
1+\gamma_{p p} \Delta+o(\Delta) & \text { if } p=q,\end{cases}
\end{aligned}
$$

where $\Delta>0$. Here, $\gamma_{p q}>0$ is the transition rate from $p$ to $q$ if $p \neq q$ while

$$
\gamma_{p p}=-\sum_{q \neq p} \gamma_{p q}
$$

We assume that the Markov chain $r(\cdot)$ is independent of the Brownian motion $W(\cdot)$. It is well known that almost every sample path of $r(\cdot)$ is a right-continuous step function with finite number of simple jumps in any finite subinterval of $\mathbb{R}_{+}:=[0,+\infty)$.

In this paper, we will consider the following hybrid BAM neural networks with reaction diffusion terms:

$$
\begin{aligned}
\frac{\partial \widetilde{u}_{i}(t, x)}{\partial t}= & \sum_{k=1}^{l} \frac{\partial}{\partial x_{k}}\left(\bar{D}_{i k}(r(t)) \frac{\partial \widetilde{u}_{i}(t, x)}{\partial x_{k}}\right) \\
& -a_{i}(r(t)) \widetilde{u}_{i}(t, x) \\
& +\sum_{j=1}^{n} c_{j i}(r(t)) \tilde{f}_{j}\left(\widetilde{v}_{j}(t, x)\right)+I_{i}, \\
\frac{\partial \widetilde{v}_{j}(t, x)}{\partial t}= & \sum_{k=1}^{l} \frac{\partial}{\partial x_{k}}\left(\bar{D}_{j k}^{*}(r(t)) \frac{\partial \widetilde{v}_{j}(t, x)}{\partial x_{k}}\right) \\
& -b_{j}(r(t)) \widetilde{v}_{j}(t, x) \\
& +\sum_{i=1}^{m} e_{i j}(r(t)) \tilde{g}_{i}\left(\tilde{u}_{i}(t, x)\right)+J_{j},
\end{aligned}
$$

where $i=1,2, \ldots, m, j=1,2, \ldots, n, t \geq t_{0} \geq 0$, $t_{0} \in \mathbb{R}_{+}$, and the initial value $r\left(t_{0}\right)=i_{0} \in \mathbb{S}$. Consider $x=\left(x_{1}, x_{2}, \ldots, x_{l}\right) \in \Omega_{0} \subset \mathbb{R}^{l} ; \Omega_{0}$ is a compact set with smooth boundary $\partial \Omega_{0}$ in space $\mathbb{R}^{l}$, and $0<\operatorname{mes} \Omega_{0}<$ $+\infty$. $\widetilde{u}(t, x)=\left(\widetilde{u}_{1}(t, x), \ldots, \widetilde{u}_{m}(t, x)\right) \in \mathbb{R}^{m}$ and $\widetilde{v}(t, x)=$ $\left(\widetilde{v}_{1}(t, x), \ldots, \widetilde{v}_{n}(t, x)\right) \in \mathbb{R}^{n} \widetilde{u}_{i}(t, x), \widetilde{v}_{j}(t, x)$, are the state of the $i$ th neurons and the $j$ th neurons at times $t$ and in space $x$, respectively. $\tilde{f}_{j}$ and $\widetilde{g}_{i}$ denote the signal functions on the $j$ th neurons and the $i$ th neurons at times $t$ and in space $x$, respectively. $I_{i}$ and $J_{j}$ denote the external input on the $i$ th neurons and the jth neurons, respectively. $a_{i}(r(t))>0$ and $b_{j}(r(t))>0$ denote the rates with which the $i$ th neurons and the $j$ th neurons will reset its potential to the resting state in isolation when disconnected from the networks and external inputs, respectively. $c_{j i}(r(t))$ and $e_{i j}(r(t))$ denote the strength of the $j$ th neurons on the $i$ th neurons and the $i$ th neurons on the $j$ th neurons, respectively. Smooth functions $\bar{D}_{i k}(r(t)):=$ $\bar{D}_{i k}(r(t), x, u) \geq 0$ and $\bar{D}_{j k}^{*}(r(t)):=\bar{D}_{j k}^{*}(r(t), x, u) \geq 0$ correspond to the transmission diffusion operator along the $i$ th neurons and the $j$ th neurons, respectively. by

The initial conditions and boundary conditions are given

$$
\begin{aligned}
& \tilde{u}_{i}\left(t_{0}, x\right)=\bar{\phi}_{i}(x), \quad x \in \Omega_{0}, t_{0} \in \mathbb{R}_{+}, i=1,2, \ldots, m, \\
& \widetilde{v}_{j}\left(t_{0}, x\right)=\bar{\psi}_{j}(x), \quad x \in \Omega_{0}, t_{0} \in \mathbb{R}_{+}, \quad j=1,2, \ldots, n, \\
& \left.\frac{\partial \tilde{u}_{i}(t, x)}{\partial \vec{n}}\right|_{\partial \Omega_{0}}=\left(\frac{\partial \tilde{u}_{i}(t, x)}{\partial x_{1}}, \ldots, \frac{\partial \widetilde{u}_{i}(t, x)}{\partial x_{l}}\right)^{T}=0,
\end{aligned}
$$




$$
\begin{gathered}
(t, x) \in\left[t_{0},+\infty\right) \times \partial \Omega_{0}, \quad i=1,2, \ldots, m, \\
\left.\frac{\partial \widetilde{v}_{j}(t, x)}{\partial \vec{n}}\right|_{\partial \Omega_{0}}=\left(\frac{\partial \widetilde{v}_{j}(t, x)}{\partial x_{1}}, \ldots, \frac{\partial \widetilde{v}_{j}(t, x)}{\partial x_{l}}\right)^{T}=0, \\
(t, x) \in\left[t_{0},+\infty\right) \times \partial \Omega_{0}, \quad j=1,2, \ldots, n .
\end{gathered}
$$

The neuron activation functions $\widetilde{f}$ and $\widetilde{g}$ are global Lipschitz continuous; that is, there exist constants $K>0$ and $L>0$, such that

$$
\begin{gathered}
\left|\widetilde{f}(\widetilde{v})-\widetilde{f}\left(\widetilde{v}^{*}\right)\right| \leq K\left|\widetilde{v}-\widetilde{v}^{*}\right|, \quad \forall \widetilde{v}, \widetilde{v}^{*} \in \mathbb{R}^{n} \\
\left|\widetilde{g}(\widetilde{u})-\widetilde{g}\left(\widetilde{u}^{*}\right)\right| \leq L\left|\widetilde{u}-\widetilde{u}^{*}\right|, \quad \forall \widetilde{u}, \widetilde{u}^{*} \in \mathbb{R}^{m} .
\end{gathered}
$$

Then, the neural networks (3) have a unique state $\left(\widetilde{u}\left(t, x ; t_{0}, \bar{\phi}(x)\right)\right.$ and $\left.\widetilde{v}\left(t, x ; t_{0}, \bar{\psi}(x)\right)\right)$ for any initial values $(\bar{\phi}(x), \bar{\psi}(x))$ (see $[26,27])$.

In addition, we assume that the neural networks (3) have an equilibrium point $u^{*}=\left(u_{1}^{*}, \ldots, u_{m}^{*}\right) \in \mathbb{R}^{m}, v^{*}=$ $\left(v_{1}^{*}, \ldots, v_{n}^{*}\right) \in \mathbb{R}^{n}$.

Let $u(t, x)=\widetilde{u}(t, x)-u^{*}, v(t, x)=\widetilde{v}(t, x)-v^{*}, f(v(t, x))=$ $\tilde{f}\left(v(t, x)+v^{*}\right)-\widetilde{f}\left(v^{*}\right), g(u(t, x))=\widetilde{g}\left(u(t, x)+u^{*}\right)-$ $\widetilde{f}\left(u^{*}\right), D_{i k}(r(t))=\bar{D}_{i k}\left(r(t), x, u(t, x)+u^{*}\right)$, and $D_{i k}^{*}(r(t))=$ $\bar{D}_{i k}^{*}\left(r(t), x, v(t, x)+v^{*}\right)$, and then (3) can be rewritten as

$$
\begin{aligned}
\frac{\partial u_{i}(t, x)}{\partial t}= & \sum_{k=1}^{l} \frac{\partial}{\partial x_{k}}\left(D_{i k}(r(t)) \frac{\partial u_{i}(t, x)}{\partial x_{k}}\right) \\
& -a_{i}(r(t)) u_{i}(t, x) \\
& +\sum_{j=1}^{n} c_{j i}(r(t)) f_{j}\left(v_{j}(t, x)\right) \\
\frac{\partial v_{j}(t, x)}{\partial t}= & \sum_{k=1}^{l} \frac{\partial}{\partial x_{k}}\left(D_{j k}^{*}(r(t)) \frac{\partial v_{j}(t, x)}{\partial x_{k}}\right) \\
& -b_{j}(r(t)) v_{j}(t, x) \\
& +\sum_{i=1}^{m} e_{i j}(r(t)) g_{i}\left(u_{i}(t, x)\right) .
\end{aligned}
$$
by

The initial conditions and boundary conditions are given

$$
\begin{gathered}
u_{i}\left(t_{0}, x\right)=\phi_{i}(x)=\bar{\phi}_{i}(x)-u_{i}^{*}, \\
x \in \Omega_{0}, t_{0} \in \mathbb{R}_{+}, \quad i=1,2, \ldots, m, \\
v_{j}\left(t_{0}, x\right)=\psi_{j}(x)=\bar{\psi}_{j}(x)-v_{j}^{*}, \\
x \in \Omega_{0}, t_{0} \in \mathbb{R}_{+}, \quad j=1,2, \ldots, n, \\
\left.\frac{\partial u_{i}(t, x)}{\partial \vec{n}}\right|_{\partial \Omega_{0}}=\left(\frac{\partial u_{i}(t, x)}{\partial x_{1}}, \ldots, \frac{\partial u_{i}(t, x)}{\partial x_{l}}\right)^{T}=0,
\end{gathered}
$$

$$
\begin{array}{r}
(t, x) \in\left[t_{0},+\infty\right) \times \partial \Omega_{0}, \quad i=1,2, \ldots, m, \\
\left.\frac{\partial v_{j}(t, x)}{\partial \vec{n}}\right|_{\partial \Omega_{0}}=\left(\frac{\partial v_{j}(t, x)}{\partial x_{1}}, \ldots, \frac{\partial v_{j}(t, x)}{\partial x_{l}}\right)^{T}=0, \\
(t, x) \in\left[t_{0},+\infty\right) \times \partial \Omega_{0}, \quad j=1,2, \ldots, n .
\end{array}
$$

Hence, the origin is an equilibrium point of (6). The stability of the equilibrium point of (3) is equivalent to the stability of the origin of the state space of (6).

From (5), we give the assumption about activations functions $f$ and $g$.

Assumption (H1). The neuron activation functions $f$ and $g$ are global Lipschitz continuous; that is, there exist constants $K>0$ and $L>0$, such that

$$
\begin{aligned}
& \left|f(v)-f\left(v^{*}\right)\right| \leq K\left|v-v^{*}\right|, \quad \forall v, v^{*} \in \mathbb{R}^{n}, f(0)=0, \\
& \left|g(u)-g\left(u^{*}\right)\right| \leq L\left|u-u^{*}\right|, \quad \forall u, u^{*} \in \mathbb{R}^{m}, g(0)=0 .
\end{aligned}
$$

We consider the following function vector space:

$$
U=\left\{\begin{array}{l}
v(t, x):\left[t_{0},+\infty\right) \times \Omega_{0} \longrightarrow \mathbb{R}^{n}, \\
v(t, x) \text { is continuous on } t \text { and } \\
\text { twice continuous differentiable on } x .
\end{array}\right.
$$

For every pair of $(v, z)$ in $U$ and every given $t \in \mathbb{R}_{+}$, define inner product for $v$ and $z$ with

$$
\langle v, z\rangle=\int_{\Omega_{0}}(v(\cdot, x))^{T} z(\cdot, x) d x \in \mathbb{R}_{+} .
$$

Obviously, it satisfies inner product axiom, and the norm can be deduced by

$$
\begin{aligned}
\|v(\cdot, x)\|_{2} & =\sqrt{\langle v(\cdot, x), v(\cdot, x)\rangle} \\
& =\sqrt{\int_{\Omega_{0}}|v(\cdot, x)|^{2} d x}=\sqrt{\sum_{i=1}^{n} \int_{\Omega_{0}}\left|v_{i}(\cdot, x)\right|^{2} d x .}
\end{aligned}
$$

Definition 1. The neural networks (6) are said to be global exponentially stable if for any $\phi, \psi$, there exist $\alpha>0$ and $\beta>0$, such that

$$
\begin{aligned}
& \left\|u\left(t, x ; t_{0}, \phi\right)\right\|_{2}^{2}+\left\|v\left(t, x ; t_{0}, \psi\right)\right\|_{2}^{2} \\
& \quad \leq \alpha\left(\|\phi\|_{2}^{2}+\|\psi\|_{2}^{2}\right) \exp \left(-\beta\left(t-t_{0}\right)\right), \quad \forall t \geq t_{0} .
\end{aligned}
$$

For the purpose of simplicity, we rewrite (6) as follows:

$$
\begin{aligned}
\frac{\partial u}{\partial t}= & \nabla \cdot(D(r(t)) \circ \nabla u)-A(r(t)) u(t, x) \\
& +C(r(t)) f(v(t, x)) \\
\frac{\partial v}{\partial t}= & \nabla \cdot\left(D^{*}(r(t)) \circ \nabla v\right)-B(r(t)) v(t, x) \\
& +E(r(t)) g(u(t, x)) .
\end{aligned}
$$



by

The initial conditions and boundary conditions are given

$$
\begin{gathered}
u\left(t_{0}, x\right)=\phi(x)=\bar{\phi}(x)-u^{*}, \quad x \in \Omega_{0}, t_{0} \in \mathbb{R}_{+}, \\
v\left(t_{0}, x\right)=\psi(x)=\bar{\psi}(x)-v^{*}, \quad x \in \Omega_{0}, t_{0} \in \mathbb{R}_{+} \\
\left.\frac{\partial u(t, x)}{\partial \vec{n}}\right|_{\partial \Omega_{0}}=\left(\frac{\partial u(t, x)}{\partial x_{1}}, \ldots, \frac{\partial u(t, x)}{\partial x_{l}}\right)^{T}=0 \\
\left.\frac{\partial v(t, x) \in\left[t_{0},+\infty\right) \times \partial \Omega_{0}}{\partial \vec{n}}\right|_{\partial \Omega_{0}}=\left(\frac{\partial v(t, x)}{\partial x_{1}}, \ldots, \frac{\partial v(t, x)}{\partial x_{l}}\right)^{T}=0 \\
(t, x) \in\left[t_{0},+\infty\right) \times \partial \Omega_{0}
\end{gathered}
$$

where

$$
\begin{aligned}
& D(r(t))=\left(D_{i k}(r(t), x, u)\right)_{m \times l} \\
& D^{*}(r(t))=\left(D_{j k}^{*}(r(t), x, v)\right)_{n \times l^{\prime}} \\
& u(t, x)=\left(u_{1}(t, x), \ldots, u_{m}(t, x)\right)^{T}, \\
& v(t, x)=\left(v_{1}(t, x), \ldots, v_{n}(t, x)\right)^{T}, \\
& \nabla u=\left(\nabla u_{1}, \ldots, \nabla u_{m}\right)^{T}, \quad \nabla v=\left(\nabla v_{1}, \ldots, \nabla v_{n}\right)^{T} \\
& \nabla u_{i}=\left(\frac{\partial u_{i}}{\partial x_{1}}, \ldots, \frac{\partial u_{i}}{\partial x_{l}}\right)^{T}, \quad \nabla v_{j}=\left(\frac{\partial v_{j}}{\partial x_{1}}, \ldots, \frac{\partial v_{j}}{\partial x_{l}}\right)^{T} \\
& A(r(t))=\operatorname{diag}\left(a_{1}(r(t)), \ldots, a_{m}(r(t))\right), \\
& B(r(t))=\operatorname{diag}\left(b_{1}(r(t)), \ldots, b_{n}(r(t))\right), \\
& C(r(t))=\left(c_{j i}(r(t))\right)_{n \times m}, \\
& E(r(t))=\left(e_{i j}(r(t))\right)_{m \times n}, \\
& f(v)=\left(f_{1}\left(v_{1}\right), \ldots, f_{n}\left(v_{n}\right)\right)^{T}, \\
& g(u)=\left(g_{1}\left(u_{1}\right), \ldots, g_{m}\left(u_{m}\right)\right)^{T}, \\
& (D(r(t)) \circ \nabla u)=\left(D_{i k}(r(t)) \frac{\partial u_{i}}{\partial x_{k}}\right), \\
& \left(D^{*}(r(t)) \circ \nabla v\right)=\left(D_{j k}^{*}(r(t)) \frac{\partial v_{j}}{\partial x_{k}}\right) .
\end{aligned}
$$

Here, o denotes Hadamard product of matrix $D$ and $\nabla u$ and $D^{*}$ and $\nabla v$.

\section{Noise Impact on Stability}

In this section, we consider the noise-induced neural networks (6) described by the stochastic partial differential equations

$$
\begin{aligned}
& \mathrm{d} \bar{u}_{i}(t, x)=\left\{\sum_{k=1}^{l} \frac{\partial}{\partial x_{k}}\left(D_{i k}(r(t)) \frac{\partial \bar{u}_{i}(t, x)}{\partial x_{k}}\right)\right. \\
&-a_{i}(r(t)) \bar{u}_{i}(t, x) \\
&\left.+\sum_{j=1}^{n} c_{j i}(r(t)) f_{j}\left(\bar{v}_{j}(t, x)\right)\right\} \mathrm{d} t \\
&+ \sigma \bar{u}_{i}(t, x) \mathrm{d} W(t), \\
& \mathrm{d} \bar{v}_{j}(t, x)=\left\{\sum_{k=1}^{l} \frac{\partial}{\partial x_{k}}\left(D_{j k}^{*}(r(t)) \frac{\partial \bar{v}_{j}(t, x)}{\partial x_{k}}\right)\right. \\
&-b_{j}(r(t)) \bar{v}_{j}(t, x) \\
&\left.+\sum_{i=1}^{m} e_{i j}(r(t)) g_{i}\left(\bar{u}_{i}(t, x)\right)\right\} \mathrm{d} t \\
&+ \sigma \bar{v}_{j}(t, x) \mathrm{d} W(t) .
\end{aligned}
$$
by

The initial conditions and boundary conditions are given

$$
\begin{gathered}
\bar{u}_{i}\left(t_{0}, x\right)=\phi_{i}(x), \quad x \in \Omega_{0}, t_{0} \in \mathbb{R}_{+}, i=1,2, \ldots, m, \\
\bar{v}_{j}\left(t_{0}, x\right)=\psi_{j}(x), \quad x \in \Omega_{0}, t_{0} \in \mathbb{R}_{+}, \quad j=1,2, \ldots, n, \\
\left.\frac{\partial \bar{u}_{i}(t, x)}{\partial \vec{n}}\right|_{\partial \Omega_{0}}=\left(\frac{\partial \bar{u}_{i}(t, x)}{\partial x_{1}}, \ldots, \frac{\partial \bar{u}_{i}(t, x)}{\partial x_{l}}\right)^{T}=0, \\
(t, x) \in\left[t_{0},+\infty\right) \times \partial \Omega_{0}, \quad i=1,2, \ldots, m, \\
\left.\frac{\partial \bar{v}_{j}(t, x)}{\partial \vec{n}}\right|_{\partial \Omega_{0}}=\left(\frac{\partial \bar{v}_{j}(t, x)}{\partial x_{1}}, \ldots, \frac{\partial \bar{v}_{j}(t, x)}{\partial x_{l}}\right)^{T}=0, \\
(t, x) \in\left[t_{0},+\infty\right) \times \partial \Omega_{0}, \quad j=1,2, \ldots, n,
\end{gathered}
$$

where $\sigma$ is the noise intensity.

We rewrite (16) as follows:

$$
\begin{aligned}
& \mathrm{d} \bar{u}(t, x)=\{\nabla \cdot(D(r(t)) \circ \nabla \bar{u})-A(r(t)) \bar{u}(t, x) \\
&+C(r(t)) f(\bar{v}(t, x))\} \mathrm{d} t+\sigma \bar{u}(t, x) \mathrm{d} W(t), \\
& \mathrm{d} \bar{v}(t, x)=\left\{\nabla \cdot\left(D^{*}(r(t)) \circ \nabla \bar{v}\right)-B(r(t)) \bar{v}(t, x)\right.
\end{aligned}
$$

$$
+E(r(t)) g(\bar{u}(t, x))\} \mathrm{d} t+\sigma \bar{v}(t, x) \mathrm{d} W(t) .
$$


For the globally exponentially stable neural networks (6), we will characterize how much stochastic noise the neural networks (16) can tolerate while maintaining global exponential stability.

Definition 2. The neural networks (16) are said to be almost surely globally exponentially stable, if for any $\phi$ and $\psi$ the Lyapunov exponent

$$
\limsup _{t \rightarrow \infty} \frac{\log \left(\left\|\bar{u}\left(t, x ; t_{0}, \phi\right)\right\|_{2}+\left\|\bar{v}\left(t, x ; t_{0}, \psi\right)\right\|_{2}\right)}{t}<0, \quad \text { a.s. }
$$

Definition 3. The neural networks (16) are said to be mean square globally exponentially stable, if, for any $\phi$ and $\psi$, the Lyapunov exponent

$$
\limsup _{t \rightarrow \infty} \frac{\log \mathbb{E}\left\{\left\|\bar{u}\left(t, x ; t_{0}, \phi\right)\right\|_{2}^{2}+\left\|\bar{v}\left(t, x ; t_{0}, \psi\right)\right\|_{2}^{2}\right\}}{t}<0,
$$

where $\left(\bar{u}\left(t, x ; t_{0}, \phi\right), \bar{v}\left(t, x ; t_{0}, \psi\right)\right)$ is the state of neural networks (16).

From the above definitions, it is clear that the almost sure global exponential stability of the neural networks (16) implies the mean square global exponential stability of the neural networks (16) (see $[26,27]$ ) but not vice versa.

Theorem 4. Under Assumption (H1), the mean square global exponential stability of neural networks (16) implies the almost sure global exponential stability of the neural networks (16).

Proof. For any $(\phi(x), \psi(x)) \quad \neq \quad(0,0)$, we denote the state $\left(\bar{u}\left(t, x ; t_{0}, \phi\right), \bar{v}\left(t, x ; t_{0}, \psi\right)\right)$ of $(16)$ as $(\bar{u}(t, x), \bar{v}(t, x))$. By Definition 3, there exist $\lambda>0$ and $C>0$, such that

$$
\begin{aligned}
& \mathbb{E}\left\{\|\bar{u}(t, x)\|_{2}^{2}+\|\bar{v}(t, x)\|_{2}^{2}\right\} \\
& \quad \leq C\left(\|\phi\|_{2}^{2}+\|\psi\|_{2}^{2}\right) e^{-\lambda\left(t-t_{0}\right)}, \quad t \geq t_{0} .
\end{aligned}
$$

Let $r(t)=p \in \mathbb{S}$. Construct average Lyapunov functional

$$
\begin{aligned}
& V(\bar{u}(t, x), \bar{v}(t, x), p) \\
& \quad=\int_{\Omega_{0}}|\bar{u}(t, x)|^{2} \mathrm{~d} x+\int_{\Omega_{0}}|\bar{v}(t, x)|^{2} \mathrm{~d} x \\
& \quad=\int_{\Omega_{0}} \sum_{i=1}^{m} \bar{u}_{i}^{2}(t, x) \mathrm{d} x+\int_{\Omega_{0}} \sum_{j=1}^{n} \bar{v}_{j}^{2}(t, x) \mathrm{d} x .
\end{aligned}
$$

Let $n=1,2, \ldots$, by Itô formula and Assumption (H1), for $t_{0}+$ $n-1 \leq t \leq t_{0}+n$,

$$
\begin{aligned}
& V(\bar{u}(t, x), \bar{v}(t, x), p) \\
& =V\left(\bar{u}\left(t_{0}+n-1, x\right), \bar{v}\left(t_{0}+n-1, x\right), p\right) \\
& +\int_{t_{0}+n-1}^{t} \int_{\Omega_{0}} 2 \bar{u}^{T}(s, x)[\nabla \cdot(D(r(s)) \circ \nabla \bar{u})-A(r(s)) \bar{u} \\
& +C(r(s)) f(\bar{v})] \mathrm{d} x \mathrm{~d} s
\end{aligned}
$$

$$
\begin{aligned}
& +\sigma^{2} \int_{t_{0}+n-1}^{t} \int_{\Omega_{0}}|\bar{u}(s, x)|^{2} \mathrm{~d} x \mathrm{~d} s \\
& +\int_{t_{0}+n-1}^{t} \int_{\Omega_{0}} 2 \bar{v}^{T}(s, x)\left[\nabla \cdot\left(D^{*}(r(s)) \circ \nabla \bar{v}\right)-B(r(s)) \bar{v}\right. \\
& +E(r(s)) g(\bar{u})] \mathrm{d} x \mathrm{~d} s \\
& +\sigma^{2} \int_{t_{0}+n-1}^{t} \int_{\Omega_{0}}|\bar{v}(s, x)|^{2} \mathrm{~d} x \mathrm{~d} s \\
& +2 \sigma \int_{t_{0}+n-1}^{t} \int_{\Omega_{0}}|\bar{u}(s, x)|^{2} \mathrm{~d} x \mathrm{~d} W(s) \\
& +2 \sigma \int_{t_{0}+n-1}^{t} \int_{\Omega_{0}}|\bar{v}(s, x)|^{2} \mathrm{~d} x \mathrm{~d} W(s) \\
& +\sum_{q=1}^{N} \gamma_{p q} \int_{t_{0}+n-1}^{t} \int_{\Omega_{0}}\left(|\bar{u}(s, x)|^{2}+|\bar{v}(s, x)|^{2}\right) \mathrm{d} x \mathrm{~d} s .
\end{aligned}
$$

By boundary condition and Gauss formula, we get

$$
\begin{aligned}
2 \int_{\Omega_{0}} \bar{u}^{T}(s, x)[\nabla \cdot(D(r(s)) \circ \nabla \bar{u})] \mathrm{d} x \\
=2 \sum_{i=1}^{m} \sum_{k=1}^{l} \int_{\Omega_{0}} \bar{u}_{i} \frac{\partial}{\partial x_{k}}\left(D_{i k}(r(s)) \frac{\partial \bar{u}_{i}}{\partial x_{k}}\right) \mathrm{d} x \\
=2 \sum_{i=1}^{m} \int_{\Omega_{0}} \nabla \cdot\left(\bar{u}_{i} D_{i k}(r(s)) \frac{\partial \bar{u}_{i}}{\partial x_{k}}\right)_{k=1}^{l} \mathrm{~d} x \\
\quad-2 \sum_{i=1}^{m} \int_{\Omega_{0}}\left(D_{i k}(r(s)) \frac{\partial \bar{u}_{i}}{\partial x_{k}}\right)_{k=1}^{l} \cdot \nabla \bar{u}_{i} \mathrm{~d} x \\
=2 \sum_{i=1}^{m} \int_{\partial \Omega_{0}}\left(\bar{u}_{i} D_{i k}(r(s)) \frac{\partial \bar{u}_{i}}{\partial x_{k}}\right)_{k=1}^{l} \mathrm{~d} x \\
\quad-2 \sum_{i=1}^{m} \sum_{k=1}^{l} \int_{\Omega_{0}} D_{i k}(r(s))\left(\frac{\partial \bar{u}_{i}}{\partial x_{k}}\right)^{2} \mathrm{~d} x \\
=-2 \sum_{i=1}^{m} \sum_{k=1}^{l} \int_{\Omega_{0}} D_{i k}(r(s))\left(\frac{\partial \bar{u}_{i}}{\partial x_{k}}\right)^{2} \mathrm{~d} x, \\
2 \int_{\Omega_{0}} \bar{v}^{T}(s, x)\left[\nabla \cdot\left(D^{*}(r(s)) \circ \nabla \bar{v}\right)\right] \mathrm{d} x \\
=2 \sum_{j=1}^{n} \sum_{k=1}^{l} \int_{\Omega_{0}} \bar{v}_{j} \frac{\partial}{\partial x_{k}}\left(D_{j k}^{*}(r(s)) \frac{\partial \bar{v}_{j}}{\partial x_{k}}\right) \mathrm{d} x \\
\quad-2 \sum_{\Omega_{0}}^{n} \int_{\Omega_{0}}\left(D_{j k}^{*}(r(s)) \frac{\partial \bar{v}_{j}}{\partial x_{k}}\right)_{k=1}^{l} \cdot \nabla \bar{v}_{j} \mathrm{~d} x \\
\left.=\bar{v}_{j} D_{j k}^{*}(r(s)) \frac{\partial \bar{v}_{j}}{\partial x_{k}}\right)_{k=1}^{l} \mathrm{~d} x
\end{aligned}
$$




$$
\begin{aligned}
= & 2 \sum_{j=1}^{n} \int_{\partial \Omega_{0}}\left(\bar{v}_{j} D_{j k}^{*}(r(s)) \frac{\partial \bar{v}_{j}}{\partial x_{k}}\right)_{k=1}^{l} \mathrm{~d} x \\
& -2 \sum_{j=1}^{n} \sum_{k=1}^{l} \int_{\Omega_{0}} D_{j k}^{*}(r(s))\left(\frac{\partial \bar{v}_{j}}{\partial x_{k}}\right)^{2} \mathrm{~d} x \\
= & -2 \sum_{j=1}^{n} \sum_{k=1}^{l} \int_{\Omega_{0}} D_{j k}^{*}(r(s))\left(\frac{\partial \bar{v}_{j}}{\partial x_{k}}\right)^{2} \mathrm{~d} x .
\end{aligned}
$$

By Hölder's inequality, we have

$$
\begin{aligned}
& \int_{\Omega_{0}} 2 \bar{u}(t, x)^{T} C(r(s)) f(\bar{v}(t, x)) \mathrm{d} x \\
& \leq \max _{p \in \mathbb{S}}\|C(p)\|\left[\int_{\Omega_{0}}|\bar{u}(t, x)|^{2} \mathrm{~d} x+K^{2} \int_{\Omega_{0}}|\bar{v}(t, x)|^{2} \mathrm{~d} x\right] \\
& \int_{\Omega_{0}} 2 \bar{v}(t, x)^{T} E(r(s)) g(\bar{u}(t, x)) \mathrm{d} x \\
& \leq \max _{p \in \mathbb{S}}\|E(p)\|\left[\int_{\Omega_{0}}|\bar{v}(t, x)|^{2} \mathrm{~d} x+L^{2} \int_{\Omega_{0}}|\bar{u}(t, x)|^{2} \mathrm{~d} x\right] .
\end{aligned}
$$

Substituting (24)-(27) into (23), we get

$$
\begin{aligned}
& V(\bar{u}(t, x), \bar{v}(t, x), p) \\
& =V\left(\bar{u}\left(t_{0}+n-1, x\right), \bar{v}\left(t_{0}+n-1, x\right), p\right) \\
& \quad+\max _{p \in \mathbb{S}}\left[2\|A(p)\|+\|C(p)\|+\|E(p)\| L^{2}+\sigma^{2}\right] \\
& \quad \times \int_{t_{0}+n-1}^{t} \int_{\Omega_{0}}|\bar{u}(s, x)|^{2} \mathrm{~d} x \mathrm{~d} s \\
& \quad+\max _{p \in \mathbb{S}}\left[2\|B(p)\|+\|E(p)\|+\|C(p)\| K^{2}+\sigma^{2}\right] \\
& \quad \times \int_{t_{0}+n-1}^{t} \int_{\Omega_{0}}|\bar{v}(s, x)|^{2} \mathrm{~d} x \mathrm{~d} s \\
& \quad+2|\sigma| \int_{t_{0}+n-1}^{t} \int_{\Omega_{0}}\left(|\bar{u}(s, x)|^{2}+|\bar{v}(s, x)|^{2}\right) \mathrm{d} x \mathrm{~d} W(s),
\end{aligned}
$$

where we use $\sum_{q=1}^{N} \gamma_{p q}=0$.

From (28), we have

$$
\begin{aligned}
& \mathbb{E}\left(\sup _{t_{0}+n-1 \leq t \leq t_{0}+n} V(\bar{u}(t, x), \bar{v}(t, x), p)\right) \\
& \leq V\left(\bar{u}\left(t_{0}+n-1, x\right), \bar{v}\left(t_{0}+n-1, x\right), p\right) \\
& \quad+C_{1} \int_{t_{0}+n-1}^{t_{0}+n} \mathbb{E} V(\bar{u}(s, x), \bar{v}(s, x), r(s)) \mathrm{d} s
\end{aligned}
$$

$$
\begin{array}{r}
+2|\sigma| \mathbb{E}\left(\sup _{t_{0}+n-1 \leq t \leq t_{0}+n} \int_{t_{0}+n-1}^{t} V(\bar{u}(s, x), \bar{v}(s, x),\right. \\
r(s)) \mathrm{d} W(s)),
\end{array}
$$

where $C_{1}=\left[2\|\widehat{A}\|+2\|\widehat{B}\|+\|\widehat{C}\|+\|\widehat{C}\| K^{2}+\|\widehat{E}\|+\|\widehat{E}\| L^{2}+2 \sigma^{2}\right]$ and $\|\widehat{A}\|=\max _{p \in \mathbb{S}}\|A(p)\|$.

On the other hand, by the Burkholder-Davis-Gundy inequality [27] and $2 \sqrt{a b} \leq(a / \varepsilon)+\varepsilon b(a>0, b>0, \varepsilon>0)$, we have

$$
\begin{aligned}
2|\sigma| & \mathbb{E}\left(\sup _{t_{0}+n-1 \leq t \leq t_{0}+n} \int_{t_{0}+n-1}^{t} V(\bar{u}(s, x), \bar{v}(s, x), r(s)) \mathrm{d} W(s)\right) \\
\leq & 4 \sqrt{2} \mathbb{E}\left(\int_{t_{0}+n-1}^{t_{0}+n} 4 \sigma^{2} V^{2}(\bar{u}(s, x), \bar{v}(s, x), r(s)) \mathrm{d} s\right)^{1 / 2} \\
\leq & 4 \sqrt{2} \mathbb{E}\left(\sup _{t_{0}+n-1 \leq t \leq t_{0}+n} V(\bar{u}(s, x), \bar{v}(s, x), r(s))\right. \\
\leq & \frac{1}{2} \mathbb{E}\left(\int_{t_{0}+n-1 \leq t \leq t_{0}+n}^{t_{0}+n} 4 \sigma^{2} V(\bar{u}(s, x), \bar{v}(s, x), r(s)) \mathrm{d} s\right)^{1 / 2} \\
& +64 \sigma^{2} \int_{t_{0}+n-1}^{t_{0}+n} \mathbb{E} V(\bar{u}(s, x), \bar{v}(s, x), r(s)) \mathrm{d} s .
\end{aligned}
$$

Substituting the above inequality into (29), we get

$$
\begin{aligned}
& \mathbb{E}\left(\sup _{t_{0}+n-1 \leq t \leq t_{0}+n} V(\bar{u}(t, x), \bar{v}(t, x), p)\right) \\
& \leq 2 \mathbb{E} V\left(\bar{u}\left(t_{0}+n-1, x\right), \bar{v}\left(t_{0}+n-1, x\right), p\right) \\
& \quad+2\left[C_{1}+64 \sigma^{2}\right] \int_{t_{0}+n-1}^{t_{0}+n} \mathbb{E} V(s) \mathrm{d} s .
\end{aligned}
$$

By induction and the mean square global exponential stability of neural networks (16),

$$
\begin{aligned}
& \mathbb{E}\left(\sup _{t_{0}+n-1 \leq t \leq t_{0}+n} V(\bar{u}(t, x), \bar{v}(t, x), p)\right) \\
& \quad \leq C\left(\|\phi\|_{2}^{2}+\|\psi\|_{2}^{2}\right)\left(2+2\left[C_{1}+64 \sigma^{2}\right]\right) e^{-\lambda(n-1)} .
\end{aligned}
$$

Let $\varepsilon \in(0, \lambda)$, by Chebyshev's inequality [27], it follows from (32) that

$$
\mathbb{P}\left\{\sup _{t_{0}+n-1 \leq t \leq t_{0}+n} V(\bar{u}(t, x), \bar{v}(t, x), p)>e^{-(\lambda-\varepsilon)(n-1)}\right\}
$$




$$
\begin{aligned}
& \leq e^{-(\lambda-\varepsilon)(n-1)} \mathbb{E}\left(\sup _{t_{0}+n-1 \leq t \leq t_{0}+n} V(\bar{u}(t, x), \bar{v}(t, x), p)\right) \\
& \leq C\left(\|\phi\|_{2}^{2}+\|\psi\|_{2}^{2}\right)\left(2+2\left[C_{1}+64 \sigma^{2}\right]\right) e^{-\varepsilon(n-1)}
\end{aligned}
$$

By Borel-Cantelli Lemma [27], for almost all $\omega \in \Omega$,

$$
\sup _{t_{0}+n-1 \leq t \leq t_{0}+n} 2 V(\bar{u}(t, x), \bar{v}(t, x), p) \leq 2 e^{-(\lambda-\varepsilon)(n-1)}
$$

holds for all but finitely many $n$. Hence, there exists an $n_{0}=$ $n_{0}(\omega)$, for all $\omega \in \Omega$, excluding a $\mathbb{P}$-null set, for the above inequality that holds whenever $n \geq n_{0}$. Consequently, for almost all $\omega \in \Omega$,

$$
\frac{\log 2 V(\bar{u}(t, x), \bar{v}(t, x), p)}{t} \leq-\frac{(\lambda-\varepsilon)(n-1)}{t_{0}+n-1}+\frac{2}{t_{0}+n-1}
$$

if $t_{0}+n-1 \leq t \leq t_{0}+n$. Therefore,

$$
\limsup _{t \rightarrow \infty} \frac{\log \left(\|\bar{u}(t, x)\|_{2}+\|\bar{v}(t, x)\|_{2}\right)}{t} \leq-\frac{(\lambda-\varepsilon)}{2} \text { a.s. }
$$

Theorem 5. Let Assumption (H1) hold and the neural networks (6) be globally exponentially stable. Then, the neural networks (16) is mean square globally exponentially stable and also almost surely globally exponentially stable, if there exist $\mu_{q}>0,(q \in \mathbb{S})$ and $|\sigma|<\bar{\sigma}$, where $\bar{\sigma}$ is a unique positive solution of the transcendental equation

$$
\begin{aligned}
\frac{4 \bar{\sigma}^{2} \alpha \widehat{\mu}}{\beta} \exp \left\{\frac{2 \Delta\left(\widehat{\mu} C_{2}+\max _{p \in \mathbb{S}} \sum_{q=1}^{N} \gamma_{p q} \mu_{q}\right)}{\breve{\mu}}\right\} \\
+2 \alpha \exp \{-\beta \Delta\}=1, \\
\Delta>\frac{\ln (2 \alpha)}{\beta}>0,
\end{aligned}
$$

where $C_{2}=\left[2\|\widehat{A}\|+2\|\widehat{B}\|+\left(1+K^{2}\right)\|\widehat{C}\|+\left(1+L^{2}\right)\|\widehat{E}\|+2 \bar{\sigma}^{2}\right]$, $\|\widehat{A}\|=\max _{p \in \mathbb{S}}\|A(p)\|$, and so forth and $\widehat{\mu}=\max _{p \in \mathbb{S}} \mu_{p}$ and $\breve{\mu}=\min _{p \in \mathbb{S}} \mu_{p}$.

Proof. For any $(\phi(x), \psi(x))$, we denote the state $\left(\bar{u}\left(t, x ; t_{0}, \phi\right), \bar{v}\left(t, x ; t_{0}, \psi\right)\right)$ of $(16)$ as $(\bar{u}(t, x), \bar{v}(t, x))$ and the state $\left(u\left(t, x ; t_{0}, \phi\right), v\left(t, x ; t_{0}, \psi\right)\right)$ of $(6)$ as $(u(t, x), v(t, x))$.

From (6) and (18) and stochastic Fubini's Theorem, we have

$$
\begin{aligned}
\int_{\Omega_{0}} & (u(t, x)-\bar{u}(t, x)) \mathrm{d} x+\int_{\Omega_{0}}(v(t, x)-\bar{v}(t, x)) \mathrm{d} x \\
= & \int_{t_{0}}^{t} \int_{\Omega_{0}} \nabla \cdot(D(r(s)) \circ \nabla(u-\bar{u})) \mathrm{d} x \mathrm{~d} s \\
& +\int_{t_{0}}^{t} \int_{\Omega_{0}}[-A(r(s))(u(s, x)-\bar{u}(s, x))
\end{aligned}
$$

$+C(r(s))(f(v(s, x))-f(\bar{v}(s, x)))] \mathrm{d} x \mathrm{~d} s$

$-\int_{t_{0}}^{t} \int_{\Omega_{0}} \sigma \bar{u}(s, x) \mathrm{d} x \mathrm{~d} W(s)$

$+\int_{t_{0}}^{t} \int_{\Omega_{0}} \nabla \cdot\left(D^{*}(r(s)) \circ \nabla(v-\bar{v})\right) \mathrm{d} x \mathrm{~d} s$

$+\int_{t_{0}}^{t} \int_{\Omega_{0}}[-B(r(s))(v(s, x)-\bar{v}(s, x))$

$+E(r(s))(g(u(s, x))-g(\bar{u}(s, x)))] \mathrm{d} x \mathrm{~d} s$

$-\int_{t_{0}}^{t} \int_{\Omega_{0}} \sigma \bar{v}(s, x) \mathrm{d} x \mathrm{~d} W(s)$.

Construct average Lyapunov functional

$\begin{aligned} V & (u(t, x), v(t, x), \bar{u}(t, x), \bar{v}(t, x), r(t)) \\ \quad & =\int_{\Omega_{0}} \mu_{r(t)}\left[|u(t, x)-\bar{u}(t, x)|^{2}+|v(t, x)-\bar{v}(t, x)|^{2}\right] \mathrm{d} x\end{aligned}$

where $\mu_{r(t)}>0$.

By applying generalized Itô formula [27], we have

$\mathrm{d} V(u(t, x), v(t, x), \bar{u}(t, x), \bar{v}(t, x), p)$

$$
\begin{aligned}
& =\int_{\Omega_{0}} 2 \mu_{p}(u(t, x)-\bar{u}(t, x))^{T}(\nabla \cdot(D(p) \circ \nabla(u-\bar{u}))) \mathrm{d} x \mathrm{~d} t \\
& +\int_{\Omega_{0}} 2 \mu_{p}(u(t, x)-\bar{u}(t, x))^{T} \\
& \quad \times[-A(p)(u(t, x)-\bar{u}(t, x)) \\
& +\int_{\Omega_{0}} \sigma^{2} \mu_{p}|\bar{u}(t, x)|^{2} \mathrm{~d} x \mathrm{~d} t \\
& +2 \int_{\Omega_{0}} \sigma(u(t, x)-\bar{u}(t, x))^{T} \bar{u}(t, x) \mathrm{d} x \mathrm{~d} W(t) \\
& +\int_{\Omega_{0}} 2 \mu_{p}(v(t, x)-\bar{v}(t, x))^{T} \\
& +\int_{\Omega_{0}} \sigma^{2} \mu_{p}|\bar{v}(t, x)|^{2} \mathrm{~d} x \mathrm{~d} t \\
& +\int_{\Omega_{0}} 2 \mu_{p}(v(t, x)-\bar{v}(t, x))^{T} \\
& \times\left[\begin{array}{l}
* \\
\quad
\end{array}\right. \\
& +E(p)(v(t, x)))] \mathrm{d} x \mathrm{~d} t
\end{aligned}
$$




$$
\begin{array}{r}
-2 \int_{\Omega_{0}} \sigma(v(t, x)-\bar{v}(t, x))^{T} \bar{v}(t, x) \mathrm{d} x \mathrm{~d} W(t) \\
+\sum_{q=1}^{N} \gamma_{p q} \mu_{q} \int_{\Omega_{0}}\left[|u(t, x)-\bar{u}(t, x)|^{2}\right. \\
\left.+|v(t, x)-\bar{v}(t, x)|^{2}\right] \mathrm{d} x .
\end{array}
$$

By boundary condition and (24), we have

$$
\begin{gathered}
2 \mu_{p} \int_{\Omega_{0}}(u(t, x)-\bar{u}(t, x))^{T}(\nabla \cdot(D(p) \circ \nabla(u-\bar{u}))) \mathrm{d} x \mathrm{~d} t \\
\quad=-2 \mu_{p} \sum_{i=1}^{m} \sum_{k=1}^{l} \int_{\Omega_{0}} D_{i k}(p)\left(\frac{\partial\left(u_{i}-\bar{u}_{i}\right)}{\partial x_{k}}\right)^{2} \mathrm{~d} x
\end{gathered}
$$

By boundary condition and (25), we have

$$
\begin{gathered}
2 \mu_{p} \int_{\Omega_{0}}(v(t, x)-\bar{v}(t, x))^{T}\left(\nabla \cdot\left(D^{*}(p) \circ \nabla(v-\bar{v})\right)\right) \mathrm{d} x \mathrm{~d} t \\
\quad=-2 \mu_{p} \sum_{j=1}^{n} \sum_{k=1}^{l} \int_{\Omega_{0}} D_{j k}^{*}(p)\left(\frac{\partial\left(v_{j}-\bar{v}_{j}\right)}{\partial x_{k}}\right)^{2} \mathrm{~d} x .
\end{gathered}
$$

By Hölder’s inequality, we get

$$
\begin{aligned}
& 2 \mu_{p} \int_{\Omega_{0}}(u(t, x)-\bar{u}(t, x))^{T} C(p) \\
& \quad \times(f(v(t, x))-f(\bar{v}(t, x))) \mathrm{d} x \\
& \leq \max _{p \in \mathbb{S}}\left(\mu_{p}\|C(p)\|\right)\left[\int_{\Omega_{0}}|u(t, x)-\bar{u}(t, x)|^{2} \mathrm{~d} x\right. \\
& \left.\quad+K^{2} \int_{\Omega_{0}}|v(t, x)-\bar{v}(t, x)|^{2} \mathrm{~d} x\right],
\end{aligned}
$$

$$
\begin{aligned}
& 2 \mu_{p} \int_{\Omega_{0}}((t, x)-\bar{v}(t, x))^{T} E(p) \\
& \times(g(u(t, x))-g(\bar{u}(t, x))) \mathrm{d} x \\
& \leq \max _{p \in \mathbb{S}}\left(\mu_{p}\|E(p)\|\right)\left[\int_{\Omega_{0}}|v(t, x)-\bar{v}(t, x)|^{2} \mathrm{~d} x\right. \\
&\left.+L^{2} \int_{\Omega_{0}}|u(t, x)-\bar{u}(t, x)|^{2} \mathrm{~d} x\right] .
\end{aligned}
$$

From (42)-(45) and Assumption (H1), we obtain that $\mathrm{d} V(u(t, x), v(t, x), \bar{u}(t, x), \bar{v}(t, x), p)$

$$
\begin{aligned}
& \leq\left(\widehat{\mu} C_{1}+\max _{p \in \mathbb{S}} \sum_{q=1}^{N} \gamma_{p q} \mu_{q}\right) \\
& \quad \times \int_{\Omega_{0}}\left(|u(t, x)-\bar{u}(t, x)|^{2}+|v(t, x)-\bar{v}(t, x)|^{2}\right) \mathrm{d} x \mathrm{~d} t
\end{aligned}
$$

$$
\begin{aligned}
& +2 \sigma^{2} \widehat{\mu} \int_{\Omega_{0}}\left(|u(t, x)|^{2}+|v(t, x)|^{2}\right) \mathrm{d} x \mathrm{~d} t \\
& -2 \int_{\Omega_{0}} \sigma(u(t, x)-\bar{u}(t, x))^{T} \bar{u}(t, x) \mathrm{d} x \mathrm{~d} W(t) \\
& -2 \int_{\Omega_{0}} \sigma(v(t, x)-\bar{v}(t, x))^{T} \bar{v}(t, x) \mathrm{d} x \mathrm{~d} W(t) .
\end{aligned}
$$

When $t \leq t_{0}+2 \Delta$, we have

$$
\begin{aligned}
& \mathbb{E} V(u(t, x), v(t, x), \bar{u}(t, x), \bar{v}(t, x), r(t)) \\
& \leq\left(\widehat{\mu} C_{1}+\max _{p \in \mathbb{S}} \sum_{q=1}^{N} \gamma_{p q} \mu_{q}\right) \\
& \quad \times \int_{t_{0}}^{t} \mathbb{E} \int_{\Omega_{0}}\left(|u(s, x)-\bar{u}(s, x)|^{2}\right. \\
& \quad+2 \sigma^{2} \widehat{\mu} \int_{t_{0}}^{t} \alpha\left(\|\phi\|_{2}^{2}+\|\psi\|_{2}^{2}\right) \exp \left(-\beta\left(s-t_{0}\right)\right) \mathrm{d} s \\
& \quad-2 \sigma \mathbb{E} \int_{t_{0}}^{t} \int_{\Omega_{0}}(u(s, x)-\bar{u}(s, x))^{T} \bar{u}(s, x) \mathrm{d} x \mathrm{~d} W(s) \\
& \quad-2 \sigma \mathbb{E} \int_{t_{0}}^{t} \int_{\Omega_{0}}(v(s, x)-\bar{v}(s, x))^{T} \bar{v}(s, x) \mathrm{d} x \mathrm{~d} W(s) .
\end{aligned}
$$

By stochastic Fubini's Theorem, we have

$$
\begin{aligned}
& \mathbb{E} \int_{t_{0}}^{t} \int_{\Omega_{0}}(u(s, x)-\bar{u}(s, x))^{T} \bar{u}(s, x) \mathrm{d} x \mathrm{~d} W(s)=0, \\
& \mathbb{E} \int_{t_{0}}^{t} \int_{\Omega_{0}}(v(s, x)-\bar{v}(s, x))^{T} \bar{v}(s, x) \mathrm{d} x \mathrm{~d} W(s)=0 .
\end{aligned}
$$

By (47), one get

$$
\begin{aligned}
& \mathbb{E} V(u(t, x), v(t, x), \bar{u}(t, x), \bar{v}(t, x), r(t)) \\
& \leq \frac{\left(\widehat{\mu} C_{1}+\max _{p \in \mathbb{S}} \sum_{q=1}^{N} \gamma_{p q} \mu_{q}\right)}{\breve{\mu}} \\
& \quad \times \int_{t_{0}}^{t} \mathbb{E} V(u(s, x), v(s, x), \bar{u}(s, x), \bar{v}(s, x), r(s)) d s \\
& \quad+\frac{2 \sigma^{2} \alpha \widehat{\mu}\left(\|\phi\|_{2}^{2}+\|\psi\|_{2}^{2}\right)}{\beta} .
\end{aligned}
$$

When $t_{0}+\Delta \leq t \leq t_{0}+2 \Delta$, by applying Gronwall's inequality, we have

$$
\begin{aligned}
\mathbb{E}( & \left.\|u(t, x)-\bar{u}(t, x)\|_{2}^{2}+\|v(t, x)-\bar{v}(t, x)\|_{2}^{2}\right) \\
& =\mathbb{E} V(u(t, x), v(t, x), \bar{u}(t, x), \bar{v}(t, x), r(t)) \\
& \leq \frac{2 \sigma^{2} \alpha \widehat{\mu}\left(\|\phi\|_{2}^{2}+\|\psi\|_{2}^{2}\right)}{\beta}
\end{aligned}
$$




$$
\begin{aligned}
& \times \exp \frac{\left(\widehat{\mu} C_{1}+\max _{p \in \mathbb{S}} \sum_{q=1}^{N} \gamma_{p q} \mu_{q}\right)}{\breve{\mu}}\left(t-t_{0}\right) \\
\leq & \sup _{t_{0} \leq t \leq t_{0}+\Delta} \mathbb{E}\left(\|\bar{u}(t, x)\|^{2}+\|\bar{v}(t, x)\|^{2}\right) \\
& \times \frac{2 \sigma^{2} \alpha \widehat{\mu}}{\beta} \exp \frac{2 \Delta\left(\widehat{\mu} C_{1}+\max _{p \in \mathbb{S}} \sum_{q=1}^{N} \gamma_{p q} \mu_{q}\right)}{\breve{\mu}} .
\end{aligned}
$$

By the global exponential stability of (6), we have

$$
\begin{aligned}
& \mathbb{E}\left(\|\bar{u}(t, x)\|_{2}^{2}+\|\bar{v}(t, x)\|_{2}^{2}\right) \\
& \leq 2 \mathbb{E}\left(\|u(t, x)-\bar{u}(t, x)\|_{2}^{2}+\|v(t, x)-\bar{v}(t, x)\|_{2}^{2}\right) \\
& \quad+2 \mathbb{E}\left(\|u(t, x)\|_{2}^{2}+\|v(t, x)\|_{2}^{2}\right) \\
& \leq \sup _{t_{0} \leq t \leq t_{0}+\Delta} \mathbb{E}\left(\|\bar{u}(t, x)\|^{2}+\|\bar{v}(t, x)\|^{2}\right) \\
& \quad \times \frac{4 \sigma^{2} \alpha \widehat{\mu}}{\beta} \exp \frac{2 \Delta\left(\widehat{\mu} C_{1}+\max _{p \in \mathbb{S}} \sum_{q=1}^{N} \gamma_{p q} \mu_{q}\right)}{\breve{\mu}} \\
& \quad+2 \alpha\left(\|\phi\|_{2}^{2}+\|\psi\|_{2}^{2}\right) \exp \left\{-\beta\left(t-t_{0}\right)\right\} .
\end{aligned}
$$

Moreover,

$$
\begin{aligned}
& \mathbb{E}\left(\|\bar{u}(t, x)\|_{2}^{2}+\|\bar{v}(t, x)\|_{2}^{2}\right) \\
& \leq\left\{\frac{4 \sigma^{2} \alpha \widehat{\mu}}{\beta} \exp \left\{\frac{2 \Delta\left(\widehat{\mu} C_{1}+\max _{p \in \mathbb{S}} \sum_{q=1}^{N} \gamma_{p q} \mu_{q}\right)}{\breve{\mu}}\right\}\right. \\
& +2 \alpha \exp \{-\beta \Delta\}\} \\
& \quad \times \sup _{t_{0} \leq t \leq t_{0}+\Delta} \mathbb{E}\left(\|\bar{u}(t, x)\|^{2}+\|\bar{v}(t, x)\|^{2}\right) .
\end{aligned}
$$

From (37), when $|\sigma|<\bar{\sigma}$, we have

$$
\begin{aligned}
& \frac{4 \sigma^{2} \alpha \widehat{\mu}}{\beta} \exp \left\{\frac{2 \Delta\left(\widehat{\mu} C_{1}+\max _{p \in \mathbb{S}} \sum_{q=1}^{N} \gamma_{p q} \mu_{q}\right)}{\breve{\mu}}\right\} \\
& +2 \alpha \exp \{-\beta \Delta\}<1 .
\end{aligned}
$$

Let

$$
\begin{aligned}
\gamma=\left(-\log \left\{\frac{4 \sigma^{2} \alpha \widehat{\mu}}{\beta}\right.\right. \\
\\
\times \exp \left\{\frac{2 \Delta\left(\widehat{\mu} C_{1}+\max _{p \in \mathbb{S}} \sum_{q=1}^{N} \gamma_{p q} \mu_{q}\right)}{\breve{\mu}}\right\}
\end{aligned}
$$

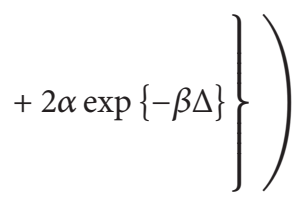$$
\times(\Delta)^{-1}>0
$$

By (52), we have

$$
\begin{aligned}
& \sup _{t_{0}+\Delta \leq t \leq t_{0}+2 \Delta} \mathbb{E}\left(\|\bar{u}(t, x)\|_{2}^{2}+\|\bar{v}(t, x)\|_{2}^{2}\right) \\
& \quad \leq \exp (-\gamma \Delta)\left(\sup _{t_{0} \leq t \leq t_{0}+\Delta} \mathbb{E}\left(\|\bar{u}(t, x)\|_{2}^{2}+\|\bar{v}(t, x)\|_{2}^{2}\right)\right) .
\end{aligned}
$$

For any positive integer $m=1,2, \ldots$, from the existence and uniqueness of the flow of (16) (see [28]), when $t \geq t_{0}+(m-$ 1) $\Delta$, we have

$$
\begin{aligned}
(\bar{u}( & \left.\left.t, x ; t_{0}, \phi\right), \bar{v}\left(t, x ; t_{0}, \psi\right)\right) \\
= & \left(\bar{u}\left(t, x ; t_{0}+(m-1) \Delta, \bar{u}\left(t_{0}+(m-1) \Delta, x ; t_{0}, \phi\right)\right),\right. \\
& \left.\bar{v}\left(t, x ; t_{0}+(m-1) \Delta, \bar{v}\left(t_{0}+(m-1) \Delta, x ; t_{0}, \psi\right)\right)\right) .
\end{aligned}
$$

From (55) and (56),

$$
\begin{aligned}
& \sup _{t_{0}+m \Delta \leq t \leq t_{0}+(m+1) \Delta} \mathbb{E}\left(\left\|\bar{u}\left(t, x ; t_{0}, \phi\right)\right\|_{2}^{2}+\left\|\bar{v}\left(t, x ; t_{0}, \psi\right)\right\|_{2}^{2}\right) \\
& =\sup _{t_{0}+(m-1) \Delta+\Delta \leq t \leq t_{0}+(m-1) \Delta+2 \Delta} \mathbb{E} \| \bar{u}\left(t, x ; t_{0}+(m-1) \Delta,\right. \\
& +\sup _{t_{0}+(m-1) \Delta+\Delta \leq t \leq t_{0}+(m-1) \Delta+2 \Delta} \mathbb{E} \| \bar{v}\left(t_{0}+(m-1) \Delta ; t_{0}+(m-1) \Delta,\right. \\
& \\
& \leq \exp (-\gamma \Delta)\left(\sup _{t_{0}+(m-1) \Delta \leq t \leq t_{0}+m \Delta} \bar{v}\left(t_{0}+(m-1) \Delta, x ; t_{0}, \psi\right)\right) \|_{2}^{2} \\
& \mathbb{E}\left(\left\|\bar{u}\left(t, x ; t_{0}, \phi\right)\right\|_{2}^{2}\right.
\end{aligned}
$$$$
\left.\left.+\left\|\bar{v}\left(t, x ; t_{0}, \psi\right)\right\|_{2}^{2}\right)\right)
$$$$
\leq \exp (-\gamma m \Delta)\left(\operatorname { s u p } _ { t _ { 0 } \leq t \leq t _ { 0 } + \Delta } \mathbb { E } \left(\left\|\bar{u}\left(t, x ; t_{0}, \phi\right)\right\|_{2}^{2}\right.\right.
$$$$
\left.\left.+\left\|\bar{v}\left(t, x ; t_{0}, \psi\right)\right\|_{2}^{2}\right)\right)
$$

Hence, for any $t \geq t_{0}+\Delta$, there exists a positive integer $m$, such that $t_{0}+m \Delta \leq t \leq t_{0}+(m+1) \Delta$, and we have 


$$
\begin{aligned}
\mathbb{E} & \left(\left\|\bar{u}\left(t, x ; t_{0}, \phi\right)\right\|_{2}^{2}+\left\|\bar{v}\left(t, x ; t_{0}, \psi\right)\right\|_{2}^{2}\right) \\
\leq & \exp (-\gamma m \Delta) \\
& \times\left(\sup _{t_{0} \leq t \leq t_{0}+\Delta} \mathbb{E}\left(\left\|\bar{u}\left(t, x ; t_{0}, \phi\right)\right\|_{2}^{2}+\left\|\bar{v}\left(t, x ; t_{0}, \psi\right)\right\|_{2}^{2}\right)\right) \\
\leq & \exp \left\{-\gamma t+\gamma t_{0}+\gamma \Delta\right\} \\
& \times\left(\sup _{t_{0} \leq t \leq t_{0}+\Delta} \mathbb{E}\left(\left\|\bar{u}\left(t, x ; t_{0}, \phi\right)\right\|_{2}^{2}+\left\|\bar{v}\left(t, x ; t_{0}, \psi\right)\right\|_{2}^{2}\right)\right) \\
\leq & C_{3} \exp \{\gamma \Delta\} \exp \left\{-\gamma\left(t-t_{0}\right)\right\},
\end{aligned}
$$

where $C_{3}=\sup _{t_{0} \leq t \leq t_{0}+\Delta} \mathbb{E}\left(\left\|\bar{u}\left(t, x ; t_{0}, \phi\right)\right\|_{2}^{2}+\left\|\bar{v}\left(t, x ; t_{0}, \psi\right)\right\|_{2}^{2}\right)$. The above inequality also holds for $t_{0} \leq t \leq t_{0}+\Delta$.

Therefore, the neural networks (16) are mean square globally exponentially stable, and by Theorem 4 , the neural networks (16) are also almost surely globally exponentially stable.

\section{Connection Weight Matrices Uncertainty and Noise Impact on Stability}

In this section, we first consider the parameter uncertainty intensity which is added to the self-feedback matrix $(A, B)^{T}$ of the neural networks (16). Then, the neural networks (16) are changed as

$$
\begin{aligned}
\mathrm{d} \bar{u}_{i}(t, x)= & \left\{\sum_{k=1}^{l} \frac{\partial}{\partial x_{k}}\left(D_{i k}(r(t)) \frac{\partial \bar{u}_{i}(t, x)}{\partial x_{k}}\right)\right. \\
& -(1+\lambda) a_{i}(r(t)) \bar{u}_{i}(t, x) \\
& \left.+\sum_{j=1}^{n} c_{j i}(r(t)) f_{j}\left(\bar{v}_{j}(t, x)\right)\right\} \mathrm{d} t \\
+ & \sigma \bar{u}_{i}(t, x) \mathrm{d} W(t) \\
\mathrm{d} \bar{v}_{j}(t, x)= & \left\{\sum_{k=1}^{l} \frac{\partial}{\partial x_{k}}\left(D_{j k}^{*}(r(t)) \frac{\partial \bar{v}_{j}(t, x)}{\partial x_{k}}\right)\right. \\
& -(1+\lambda) b_{j}(r(t)) \bar{v}_{j}(t, x) \\
& \left.+\sum_{i=1}^{m} e_{i j}(r(t)) g_{i}\left(\bar{u}_{i}(t, x)\right)\right\} \mathrm{d} t \\
+ & \sigma \bar{v}_{j}(t, x) \mathrm{d} W(t) .
\end{aligned}
$$
by

The initial conditions and boundary conditions are given

$$
\begin{gathered}
\bar{u}_{i}\left(t_{0}, x\right)=\phi_{i}(x), \quad x \in \Omega_{0}, t_{0} \in \mathbb{R}_{+}, i=1,2, \ldots, m, \\
\bar{v}_{j}\left(t_{0}, x\right)=\psi_{j}(x), \quad x \in \Omega_{0}, t_{0} \in \mathbb{R}_{+}, \quad j=1,2, \ldots, n, \\
\left.\frac{\partial \bar{u}_{i}(t, x)}{\partial \vec{n}}\right|_{\partial \Omega_{0}}=\left(\frac{\partial \bar{u}_{i}(t, x)}{\partial x_{1}}, \ldots, \frac{\partial \bar{u}_{i}(t, x)}{\partial x_{l}}\right)^{T}=0,
\end{gathered}
$$

$$
\begin{gathered}
(t, x) \in\left[t_{0},+\infty\right) \times \partial \Omega_{0}, \quad i=1,2, \ldots, m, \\
\left.\frac{\partial \bar{v}_{j}(t, x)}{\partial \vec{n}}\right|_{\partial \Omega_{0}}=\left(\frac{\partial \bar{v}_{j}(t, x)}{\partial x_{1}}, \ldots, \frac{\partial \bar{v}_{j}(t, x)}{\partial x_{l}}\right)^{T}=0, \\
(t, x) \in\left[t_{0},+\infty\right) \times \partial \Omega_{0}, \quad j=1,2, \ldots, n,
\end{gathered}
$$

where $\lambda$ is the self-feedback matrix $(A, B)^{T}$ uncertainty intensity and $\sigma$ is the noise intensity.

We rewrite (59) as follows:

$$
\begin{aligned}
\mathrm{d} \bar{u}(t, x)=\{\nabla & \cdot(D(r(t)) \circ \nabla \bar{u})-(1+\lambda) A(r(t)) \bar{u}(t, x) \\
+ & +(r(t)) f(\bar{v}(t, x))\} \mathrm{d} t+\sigma \bar{u}(t, x) \mathrm{d} W(t) \\
\mathrm{d} \bar{v}(t, x)=\{\nabla & \cdot\left(D^{*}(r(t)) \circ \nabla \bar{v}\right)-(1+\lambda) B(r(t)) \bar{v}(t, x) \\
& +E(r(t)) g(\bar{u}(t, x))\} \mathrm{d} t+\sigma \bar{v}(t, x) \mathrm{d} W(t) .
\end{aligned}
$$

For the global exponential stability of neural networks (6), we will characterize how much the intensity of both the self-feedback matrix $(A, B)^{T}$ uncertainty and stochastic noise the stochastic neural networks (59) can tolerate while maintaining global exponential stability.

Theorem 6. Let Assumption (H1) hold and let the neural networks (6) be globally exponentially stable. Then, the neural networks (59) are mean square globally exponential stability and also almost sure globally exponential stability, if there exists $\mu_{q}>0,(q \in \mathbb{S})$, and $(\lambda, \sigma)$ is in the inner of the closed curve described by the following transcendental equation:

$$
\begin{gathered}
\frac{4 \widehat{\mu}\left(\sigma^{2}+\lambda^{2}(\|\widehat{A}\|+\|\widehat{B}\|)\right) \alpha}{\beta} \\
\times \exp \left\{\frac{2 \Delta\left(\widehat{\mu} C_{4}+\max _{p \in \mathbb{S}} \sum_{q=1}^{N} \gamma_{p q} \mu_{q}\right)}{\breve{\mu}}\right\}, \\
+2 \alpha \exp \{-\beta \Delta\}=1, \\
\Delta>\frac{\ln (2 \alpha)}{\beta}>0,
\end{gathered}
$$

where $C_{4}=\left[\left(3+2 \lambda^{2}\right)(\|\widehat{A}\|+\|\widehat{B}\|)+\left(1+K^{2}\right)\|\widehat{C}\|+\left(1+L^{2}\right)\|\widehat{E}\|+\right.$ $\left.2 \sigma^{2}\right],\|\widehat{A}\|=\max _{p \in \mathbb{S}}\|A(p)\|$, and so forth and $\widehat{\mu}=\max _{p \in \mathbb{S}} \mu_{p}$ and $\breve{\mu}=\min _{p \in \mathbb{S}} \mu_{p}$.

Proof. For any $(\phi(x), \psi(x))$, we denote the state $\left(\bar{u}\left(t, x ; t_{0}, \phi\right), \bar{v}\left(t, x ; t_{0}, \psi\right)\right)$ of $(59)$ as $(\bar{u}(t, x), \bar{v}(t, x))$ and the state $\left(u\left(t, x ; t_{0}, \phi\right), v\left(t, x ; t_{0}, \psi\right)\right)$ of $(6)$ as $(u(t, x), v(t, x))$.

From (6) and (61) and stochastic Fubini's Theorem, we have

$$
\begin{gathered}
\int_{\Omega_{0}}(u(t, x)-\bar{u}(t, x)) \mathrm{d} x+\int_{\Omega_{0}}(v(t, x)-\bar{v}(t, x)) \mathrm{d} x \\
=\int_{t_{0}}^{t} \int_{\Omega_{0}} \nabla \cdot(D(r(s)) \circ \nabla(u-\bar{u})) \mathrm{d} x \mathrm{~d} s
\end{gathered}
$$




$$
\begin{aligned}
& +\int_{t_{0}}^{t} \int_{\Omega_{0}}[-A(r(s))(u(s, x)-\bar{u}(s, x)) \\
& +C(r(s))(f(v(s, x))-f(\bar{v}(s, x)))] \mathrm{d} x \mathrm{~d} s \\
& -\int_{t_{0}}^{t} \int_{\Omega_{0}} \sigma \bar{u}(s, x) \mathrm{d} x \mathrm{~d} W(s) \\
& +\int_{t_{0}}^{t} \int_{\Omega_{0}} \lambda A(r(s)) \bar{u}(s, x) \mathrm{d} x \mathrm{~d} s \\
& +\int_{t_{0}}^{t} \int_{\Omega_{0}} \nabla \cdot\left(D^{*}(r(s)) \circ \nabla(v-\bar{v})\right) \mathrm{d} x \mathrm{~d} s \\
& +\int_{t_{0}}^{t} \int_{\Omega_{0}}[-B(r(s))(v(s, x)-\bar{v}(s, x)) \\
& -\int_{t_{0}}^{t} \int_{\Omega_{0}} \sigma \bar{v}(s, x) \mathrm{d} x \mathrm{~d} W(s) \\
& +\int_{t_{0}}^{t} \int_{\Omega_{0}} \lambda B(r(s)) \bar{v}(s, x) \mathrm{d} x \mathrm{~d} s .
\end{aligned}
$$

\section{Construct the average Lyapunov functional}

$$
\begin{aligned}
V & (u(t, x), v(t, x), \bar{u}(t, x), \bar{v}(t, x), r(t)) \\
\quad & =\int_{\Omega_{0}} \mu_{r(t)}\left[|u(t, x)-\bar{u}(t, x)|^{2}+|v(t, x)-\bar{v}(t, x)|^{2}\right] \mathrm{d} x,
\end{aligned}
$$

where $\mu_{r(t)}>0$.

By applying generalized Itô formula [27], we have

$$
\begin{aligned}
& \left.\mathrm{d} V(u(t, x), v(t, x), \bar{u}(t, x), \bar{v}(t, x), p)\right|_{(21)} \\
& =\int_{\Omega_{0}} 2 \mu_{p}(u(t, x)-\bar{u}(t, x))^{T}(\nabla \cdot(D(p) \circ \nabla(u-\bar{u}))) \mathrm{d} x \mathrm{~d} t \\
& +\int_{\Omega_{0}} 2 \mu_{p}(u(t, x)-\bar{u}(t, x))^{T} \\
& \quad \times[-A(p)(u(t, x)-\bar{u}(t, x)) \\
& \quad+C(p)(f(v(t, x))-f(\bar{v}(t, x))) \\
& \quad+\lambda A(p) \bar{u}(t, x)] \mathrm{d} x \mathrm{~d} t \\
& +\int_{\Omega_{0}} \sigma^{2} \mu_{p}|\bar{u}(t, x)|^{2} \mathrm{~d} x \mathrm{~d} t \\
& -2 \int_{\Omega_{0}} \sigma(u(t, x)-\bar{u}(t, x))^{T} \bar{u}(t, x) \mathrm{d} x \mathrm{~d} W(t) \\
& +\int_{\Omega_{0}} 2 \mu_{p}(v(t, x)-\bar{v}(t, x))^{T} \\
& \quad \times\left(\nabla \cdot\left(D^{*}(p) \circ \nabla(v-\bar{v})\right)\right) \mathrm{d} x \mathrm{~d} t \\
& +\int_{\Omega_{0}} 2 \mu_{p}(v(t, x)-\bar{v}(t, x))^{T}
\end{aligned}
$$

$$
\begin{gathered}
\times[-B(p)(v(t, x)-\bar{v}(t, x)) \\
+E(p)(g(u(t, x))-g(\bar{u}(t, x))) \\
+\lambda B(p) \bar{v}(t, x)] \mathrm{d} x \mathrm{~d} t \\
+\int_{\Omega_{0}} \sigma^{2} \mu_{p}|\bar{v}(t, x)|^{2} \mathrm{~d} x \mathrm{~d} t \\
-2 \int_{\Omega_{0}} \sigma(v(t, x)-\bar{v}(t, x))^{T} \bar{v}(t, x) \mathrm{d} x \mathrm{~d} W(t) \\
+\sum_{q=1}^{N} \gamma_{p q} \mu_{q} \int_{\Omega_{0}}\left[|u(t, x)-\bar{u}(t, x)|^{2}\right. \\
\left.+|v(t, x)-\bar{v}(t, x)|^{2}\right] \mathrm{d} x .
\end{gathered}
$$

By Hölder's inequality, we get

$$
\begin{aligned}
& \int_{\Omega_{0}} 2 \mu_{p}(u(t, x)-\bar{u}(t, x))^{T} \lambda A(p) \bar{u}(t, x) \mathrm{d} x \\
& \leq \max _{p \in \mathbb{S}}\left\|\mu_{p} A(p)\right\|\left[\int_{\Omega_{0}}|u(t, x)-\bar{u}(t, x)|^{2} \mathrm{~d} x\right.
\end{aligned}
$$

$$
\left.+\lambda^{2} \int_{\Omega_{0}}|\bar{u}(t, x)|^{2} \mathrm{~d} x\right]
$$

$$
=\max _{p \in \mathbb{S}}\left\|\mu_{p} A(p)\right\|\left[\int_{\Omega_{0}}|u(t, x)-\bar{u}(t, x)|^{2} \mathrm{~d} x\right.
$$$$
\left.+\lambda^{2} \int_{\Omega_{0}}|u(t, x)-\bar{u}(t, x)-u(t, x)|^{2} \mathrm{~d} x\right]
$$$$
\leq \max _{p \in \mathbb{S}}\left\|\mu_{p} A(p)\right\|\left[\left(1+2 \lambda^{2}\right) \int_{\Omega_{0}}|u(t, x)-\bar{u}(t, x)|^{2} \mathrm{~d} x\right.
$$

$$
\left.+2 \lambda^{2} \int_{\Omega_{0}}|u(t, x)|^{2} \mathrm{~d} x\right]
$$

$$
\int_{\Omega_{0}} 2 \mu_{p}(v(t, x)-\bar{v}(t, x))^{T} \lambda B(p) \bar{v}(t, x) \mathrm{d} x
$$$$
\leq \max _{p \in \mathbb{S}}\left\|\mu_{p} B(p)\right\|\left[\int_{\Omega_{0}}|v(t, x)-\bar{v}(t, x)|^{2} \mathrm{~d} x\right.
$$$$
\left.+\lambda^{2} \int_{\Omega_{0}}|\bar{v}(t, x)|^{2} \mathrm{~d} x\right]
$$$$
=\max _{p \in \mathbb{S}}\left\|\mu_{p} B(p)\right\|\left[\int_{\Omega_{0}}|v(t, x)-\bar{v}(t, x)|^{2} \mathrm{~d} x\right.
$$

$$
\left.+\lambda^{2} \int_{\Omega_{0}}|v(t, x)-\bar{v}(t, x)-v(t, x)|^{2} \mathrm{~d} x\right]
$$

$\leq \max _{p \in \mathbb{S}}\left\|\mu_{p} B(p)\right\|\left[\left(1+2 \lambda^{2}\right) \int_{\Omega_{0}}|v(t, x)-\bar{v}(t, x)|^{2} \mathrm{~d} x\right.$

$$
\left.+2 \lambda^{2} \int_{\Omega_{0}}|v(t, x)|^{2} \mathrm{~d} x\right] .
$$


From (42), (43), and (67) and Assumption (H1), we obtain that

$$
\begin{aligned}
& \mathrm{d} V(u(t, x), v(t, x), \bar{u}(t, x), \bar{v}(t, x), p) \\
& \leq\left(\widehat{\mu} C_{4}+\max _{p \in \mathbb{S}} \sum_{q=1}^{N} \gamma_{p q} \mu_{q}\right) \\
& \quad \times \int_{\Omega_{0}}\left(|u(t, x)-\bar{u}(t, x)|^{2}\right. \\
& \left.\quad+|v(t, x)-\bar{v}(t, x)|^{2}\right) \mathrm{d} x \mathrm{~d} t \\
& \quad+2 \widehat{\mu}\left(\sigma^{2}+\lambda^{2}(\|\widehat{A}\|+\|\widehat{B}\|)\right) \\
& \quad \times \int_{\Omega_{0}}\left(|u(t, x)|^{2}+|v(t, x)|^{2}\right) \mathrm{d} x \mathrm{~d} t \\
& \quad-2 \int_{\Omega_{0}} \sigma(u(t, x)-\bar{u}(t, x))^{T} \bar{u}(t, x) \mathrm{d} x \mathrm{~d} W(t) \\
& \quad-2 \int_{\Omega_{0}} \sigma(v(t, x)-\bar{v}(t, x))^{T} \bar{v}(t, x) \mathrm{d} x \mathrm{~d} W(t) .
\end{aligned}
$$

When $t \leq t_{0}+2 \Delta$, we have

$$
\begin{aligned}
& \mathbb{E} V(u(t, x), v(t, x), \bar{u}(t, x), \bar{v}(t, x), r(t)) \\
& \leq\left(\widehat{\mu} C_{4}+\max _{p \in \mathbb{S}} \sum_{q=1}^{N} \gamma_{p q} \mu_{q}\right) \\
& \quad \times \int_{t_{0}}^{t} \mathbb{E} \int_{\Omega_{0}}\left(|u(s, x)-\bar{u}(s, x)|^{2}\right. \\
& \left.\quad+|v(s, x)-\bar{v}(s, x)|^{2}\right) \mathrm{d} x \mathrm{~d} s \\
& +2 \widehat{\mu}\left(\sigma^{2}+\lambda^{2}(\|\widehat{A}\|+\|\widehat{B}\|)\right) \\
& \quad \times \int_{t_{0}}^{t} \alpha\left(\|\phi\|_{2}^{2}+\|\psi\|_{2}^{2}\right) \exp \left(-\beta\left(s-t_{0}\right)\right) \mathrm{d} s \\
& -2 \sigma \mathbb{E} \int_{t_{0}}^{t} \int_{\Omega_{0}}(u(s, x)-\bar{u}(s, x))^{T} \bar{u}(s, x) \mathrm{d} x \mathrm{~d} W(s) \\
& -2 \sigma \mathbb{E} \int_{t_{0}}^{t} \int_{\Omega_{0}}(v(s, x)-\bar{v}(s, x))^{T} \bar{v}(s, x) \mathrm{d} x \mathrm{~d} W(s) .
\end{aligned}
$$

By stochastic Fubini’s Theorem, we have

$$
\begin{aligned}
& \mathbb{E} \int_{t_{0}}^{t} \int_{\Omega_{0}}(u(s, x)-\bar{u}(s, x))^{T} \bar{u}(s, x) \mathrm{d} x \mathrm{~d} W(s)=0, \\
& \mathbb{E} \int_{t_{0}}^{t} \int_{\Omega_{0}}(v(s, x)-\bar{v}(s, x))^{T} \bar{v}(s, x) \mathrm{d} x \mathrm{~d} W(s)=0 .
\end{aligned}
$$

By (69), one get

$$
\begin{aligned}
& \mathbb{E} V(u(t, x), v(t, x), \bar{u}(t, x), \bar{v}(t, x), r(t)) \\
& \leq \frac{\left(\widehat{\mu} C_{4}+\max _{p \in \mathbb{S}} \sum_{q=1}^{N} \gamma_{p q} \mu_{q}\right)}{\breve{\mu}} \\
& \quad \times \int_{t_{0}}^{t} \mathbb{E} V(u(s, x), v(s, x), \bar{u}(s, x), \bar{v}(s, x), r(s)) d s \\
& \quad+\frac{2 \widehat{\mu}\left(\sigma^{2}+\lambda^{2}(\|\widehat{A}\|+\|\widehat{B}\|)\right) \alpha\left(\|\phi\|_{2}^{2}+\|\psi\|_{2}^{2}\right)}{\beta} .
\end{aligned}
$$

When $t_{0}+\Delta \leq t \leq t_{0}+2 \Delta$, by applying Gronwall's inequality, we have

$$
\begin{aligned}
\mathbb{E}\left(\|u(t, x)-\bar{u}(t, x)\|_{2}^{2}+\|v(t, x)-\bar{v}(t, x)\|_{2}^{2}\right) \\
=\mathbb{E} V(u(t, x), v(t, x), \bar{u}(t, x), \bar{v}(t, x), r(t)) \\
\leq \frac{2 \widehat{\mu}\left(\sigma^{2}+\lambda^{2}(\|\widehat{A}\|+\|\widehat{B}\|)\right) \alpha\left(\|\phi\|_{2}^{2}+\|\psi\|_{2}^{2}\right)}{\beta} \\
\quad \times \exp \frac{\left(\widehat{\mu} C_{4}+\max _{p \in \mathbb{S}} \sum_{q=1}^{N} \gamma_{p q} \mu_{q}\right)}{\breve{\mu}}\left(t-t_{0}\right) \\
\leq \sup _{t_{0} \leq t \leq t_{0}+\Delta} \mathbb{E}\left(\|\bar{u}(t, x)\|^{2}+\|\bar{v}(t, x)\|^{2}\right) \\
\quad \times \frac{2 \widehat{\mu}\left(\sigma^{2}+\lambda^{2}(\|\widehat{A}\|+\|\widehat{B}\|)\right) \alpha}{\beta} \\
\quad \times \exp \frac{2 \Delta\left(\widehat{\mu} C_{4}+\max _{p \in \mathbb{S}} \sum_{q=1}^{N} \gamma_{p q} \mu_{q}\right)}{\breve{\mu}} .
\end{aligned}
$$

By the global exponential stability of (6), we have

$$
\begin{aligned}
& \mathbb{E}\left(\|\bar{u}(t, x)\|_{2}^{2}+\|\bar{v}(t, x)\|_{2}^{2}\right) \\
& \leq 2 \mathbb{E}\left(\|u(t, x)-\bar{u}(t, x)\|_{2}^{2}+\|v(t, x)-\bar{v}(t, x)\|_{2}^{2}\right) \\
& +2 \mathbb{E}\left(\|u(t, x)\|_{2}^{2}+\|v(t, x)\|_{2}^{2}\right) \\
& \leq \sup _{t_{0} \leq t \leq t_{0}+\Delta} \mathbb{E}\left(\|\bar{u}(t, x)\|^{2}+\|\bar{v}(t, x)\|^{2}\right) \\
& \quad \times \frac{4 \widehat{\mu}\left(\sigma^{2}+\lambda^{2}(\|\widehat{A}\|+\|\widehat{B}\|)\right) \alpha}{\beta} \\
& \quad \times \exp \frac{2 \Delta\left(\widehat{\mu} C_{4}+\max _{p \in \mathbb{S}} \sum_{q=1}^{N} \gamma_{p q} \mu_{q}\right)}{\breve{\mu}} \\
& \quad+2 \alpha\left(\|\phi\|_{2}^{2}+\|\psi\|_{2}^{2}\right) \exp \left\{-\beta\left(t-t_{0}\right)\right\} .
\end{aligned}
$$


Moreover,

$$
\begin{aligned}
& \mathbb{E}\left(\|\bar{u}(t, x)\|_{2}^{2}+\|\bar{v}(t, x)\|_{2}^{2}\right) \\
& \leq\left\{\frac{4 \widehat{\mu}\left(\sigma^{2}+\lambda^{2}(\|\widehat{A}\|+\|\widehat{B}\|)\right) \alpha}{\beta}\right. \\
& \quad \times \exp \left\{\frac{2 \Delta\left(\widehat{\mu} C_{4}+\max _{p \in \mathbb{S}} \sum_{q=1}^{N} \gamma_{p q} \mu_{q}\right)}{\breve{\mu}}\right\} \\
& +2 \alpha \exp \{-\beta \Delta\}\} \\
& \times \sup _{t_{0} \leq t \leq t_{0}+\Delta} \mathbb{E}\left(\|\bar{u}(t, x)\|^{2}+\|\bar{v}(t, x)\|^{2}\right) .
\end{aligned}
$$

From $(62)$, when $(\lambda, \sigma)$ is in the inner of the closed curve described by the transcendental equation, we have

$$
\begin{aligned}
& \frac{4 \widehat{\mu}\left(\sigma^{2}+\lambda^{2}(\|\widehat{A}\|+\|\widehat{B}\|)\right) \alpha}{\beta} \\
& \quad \times \exp \left\{\frac{2 \Delta\left(\widehat{\mu} C_{4}+\max _{p \in \mathbb{S}} \sum_{q=1}^{N} \gamma_{p q} \mu_{q}\right)}{\breve{\mu}}\right\} \\
& +2 \alpha \exp \{-\beta \Delta\}<1 .
\end{aligned}
$$

Let

$$
\begin{aligned}
\gamma=\left(-\log \left\{\frac{4 \widehat{\mu}\left(\sigma^{2}+\lambda^{2}(\|\widehat{A}\|+\|\widehat{B}\|)\right) \alpha}{\beta}\right.\right. \\
\\
\quad \times \exp \left\{\frac{2 \Delta\left(\widehat{\mu} C_{4}+\max _{p \in \mathbb{S}} \sum_{q=1}^{N} \gamma_{p q} \mu_{q}\right)}{\breve{\mu}}\right\} \\
+2 \alpha \exp \{-\beta \Delta\}\}(\Delta)^{-1}>0 .
\end{aligned}
$$

By (74), we have

$$
\begin{aligned}
& \sup _{t_{0}+\Delta \leq t \leq t_{0}+2 \Delta} \mathbb{E}\left(\|\bar{u}(t, x)\|_{2}^{2}+\|\bar{v}(t, x)\|_{2}^{2}\right) \\
& \quad \leq \exp (-\gamma \Delta)\left(\sup _{t_{0} \leq t \leq t_{0}+\Delta} \mathbb{E}\left(\|\bar{u}(t, x)\|_{2}^{2}+\|\bar{v}(t, x)\|_{2}^{2}\right)\right) .
\end{aligned}
$$

Similar to the proof of Theorem 5, we can prove that the neural networks (59) are mean square globally exponentially stable and also almost surely globally exponentially stable.

To continue, we consider the parameter uncertainty intensity which is added to the connection weight matrix $(C, E)^{T}$ of the neural networks (16). Then, the neural networks (16) are changed as

$$
\begin{aligned}
& \mathrm{d} \bar{u}_{i}(t, x)=\left\{\sum_{k=1}^{l} \frac{\partial}{\partial x_{k}}\left(D_{i k}(r(t)) \frac{\partial \bar{u}_{i}(t, x)}{\partial x_{k}}\right)\right. \\
&-a_{i}(r(t)) \bar{u}_{i}(t, x) \\
&\left.+\sum_{j=1}^{n}(1+\delta) c_{j i}(r(t)) f_{j}\left(\bar{v}_{j}(t, x)\right)\right\} \mathrm{d} t \\
&+\sigma \bar{u}_{i}(t, x) \mathrm{d} W(t), \\
& \mathrm{d} \bar{v}_{j}(t, x)=\left\{\sum_{k=1}^{l} \frac{\partial}{\partial x_{k}}\left(D_{j k}^{*}(r(t)) \frac{\partial \bar{v}_{j}(t, x)}{\partial x_{k}}\right)\right. \\
&-b_{j}(r(t)) \bar{v}_{j}(t, x) \\
&\left.+\sum_{i=1}^{m}(1+\delta) e_{i j}(r(t)) g_{i}\left(\bar{u}_{i}(t, x)\right)\right\} \mathrm{d} t \\
&+ \sigma \bar{v}_{j}(t, x) \mathrm{d} W(t) .
\end{aligned}
$$
by

The initial conditions and boundary conditions are given

$$
\begin{gathered}
\bar{u}_{i}\left(t_{0}, x\right)=\phi_{i}(x), \quad x \in \Omega_{0}, t_{0} \in \mathbb{R}_{+}, i=1,2, \ldots, m, \\
\bar{v}_{j}\left(t_{0}, x\right)=\psi_{j}(x), \quad x \in \Omega_{0}, t_{0} \in \mathbb{R}_{+}, j=1,2, \ldots, n, \\
\left.\frac{\partial \bar{u}_{i}(t, x)}{\partial \vec{n}}\right|_{\partial \Omega_{0}}=\left(\frac{\partial \bar{u}_{i}(t, x)}{\partial x_{1}}, \ldots, \frac{\partial \bar{u}_{i}(t, x)}{\partial x_{l}}\right)^{T}=0, \\
(t, x) \in\left[t_{0},+\infty\right) \times \partial \Omega_{0}, \quad i=1,2, \ldots, m, \\
\left.\frac{\partial \bar{v}_{j}(t, x)}{\partial \vec{n}}\right|_{\partial \Omega_{0}}=\left(\frac{\partial \bar{v}_{j}(t, x)}{\partial x_{1}}, \ldots, \frac{\partial \bar{v}_{j}(t, x)}{\partial x_{l}}\right)^{T}=0, \\
(t, x) \in\left[t_{0},+\infty\right) \times \partial \Omega_{0}, \quad j=1,2, \ldots, n,
\end{gathered}
$$

where $\delta$ is the connection weight matrix $(C, E)^{T}$ uncertainty intensity and $\sigma$ is the noise intensity.

We rewrite (78) as follows:

$$
\begin{aligned}
\mathrm{d} \bar{u}(t, x)= & \{\nabla \cdot(D(r(t)) \circ \nabla \bar{u})-A(r(t)) \bar{u}(t, x) \\
& +(1+\delta) C(r(t)) f(\bar{v}(t, x))\} \mathrm{d} t
\end{aligned}
$$




$$
\begin{aligned}
& +\sigma \bar{u}(t, x) \mathrm{d} W(t), \\
\mathrm{d} \bar{v}(t, x)= & \left\{\nabla \cdot\left(D^{*}(r(t)) \circ \nabla \bar{v}\right)-B(r(t)) \bar{v}(t, x)\right. \\
& +(1+\delta) E(r(t)) g(\bar{u}(t, x))\} \mathrm{d} t \\
& +\sigma \bar{v}(t, x) \mathrm{d} W(t) .
\end{aligned}
$$

For the global exponential stability of neural networks (6), we will characterize how much the intensity of both the connection weight matrix $(C, E)^{T}$ uncertainty and stochastic noise the stochastic neural networks (78) can tolerate while maintaining global exponential stability.

Theorem 7. Let Assumption (H1) hold and let the neural networks (6) be global exponential stability. Then, the neural networks (78) are mean square globally exponentially stable and also almost surely globally exponentially stable, if there exists $\mu_{q}>0,(q \in \mathbb{S})$, and $(\delta, \sigma)$ is in the inner of the closed curve described by the following transcendental equation:

$$
\begin{gathered}
\frac{4 \widehat{\mu}\left(\sigma^{2}+\delta^{2}\left(K^{2}\|\widehat{C}\|+L^{2}\|\widehat{E}\|\right)\right) \alpha}{\beta} \\
\times \exp \left\{\frac{2 \Delta\left(\widehat{\mu} C_{5}+\max _{p \in \mathbb{S}} \sum_{q=1}^{N} \gamma_{p q} \mu_{q}\right)}{\breve{\mu}}\right\} \\
+2 \alpha \exp \{-\beta \Delta\}=1, \\
\Delta>\frac{\ln (2 \alpha)}{\beta}>0,
\end{gathered}
$$

where $C_{5}=\left[2(\|\widehat{A}\|+\|\widehat{B}\|)+\left(2+\left(1+2 \delta^{2}\right) K^{2}\right)\|\widehat{C}\|+(2+(1+\right.$ $\left.\left.\left.2 \delta^{2}\right) L^{2}\right)\|\widehat{E}\|+2 \sigma^{2}\right],\|\widehat{A}\|=\max _{p \in \mathbb{S}}\|A(p)\|$, and so forth and $\widehat{\mu}=\max _{p \in \mathbb{S}} \mu_{p}$ and $\breve{\mu}=\min _{p \in \mathbb{S}} \mu_{p}$.

The proof is similar to the proof of Theorem 6 .

\section{Illustrate Example}

Example 1. Consider hybrid BAM neural networks with reaction diffusion terms

$$
\begin{aligned}
\frac{\partial u(t, x)}{\partial t}= & D(r(t)) \frac{\partial^{2} u(t, x)}{\partial x^{2}}-a(r(t)) u(t, x) \\
& +c(r(t)) f(v(t, x)), \\
\frac{\partial v(t, x)}{\partial t}= & D_{k}^{*}(r(t)) \frac{\partial^{2} v(t, x)}{\partial x^{2}}-b(r(t)) v(t, x) \\
& +e(r(t)) g(u(t, x)) .
\end{aligned}
$$
by

The initial conditions and boundary conditions are given

$$
\begin{gathered}
u(0, x)=\sin (x), \quad x \in[-5,5], \\
v(0, x)=\cos (x)-1, \quad x \in[-5,5],
\end{gathered}
$$

$$
\begin{gathered}
\left.\frac{\partial u(t, x)}{\partial \vec{n}}\right|_{\partial \Omega_{0}}=\frac{\partial u(t, x)}{\partial x}=0, \quad(t, x) \in\left[t_{0},+\infty\right) \times \partial \Omega_{0}, \\
\left.\frac{\partial v(t, x)}{\partial \vec{n}}\right|_{\partial \Omega_{0}}=\frac{\partial v(t, x)}{\partial x}=0, \quad(t, x) \in\left[t_{0},+\infty\right) \times \partial \Omega_{0},
\end{gathered}
$$

where

$$
\begin{aligned}
& \Gamma=\left(\begin{array}{cc}
-2 & 2 \\
1 & -1
\end{array}\right), \\
&\left(\begin{array}{cc}
D(1) & 0 \\
0 & D^{*}(1)
\end{array}\right)=\left(\begin{array}{cc}
0.002 & 0 \\
0 & 0.003
\end{array}\right), \\
&\left(\begin{array}{cc}
D(2) & 0 \\
0 & D^{*}(2)
\end{array}\right)=\left(\begin{array}{cc}
0.001 & 0 \\
0 & 0.002
\end{array}\right), \\
&\left(\begin{array}{cc}
a(1) & 0 \\
0 & b(1)
\end{array}\right)=\left(\begin{array}{cc}
0.2 & 0 \\
0 & 0.3
\end{array}\right), \\
&\left(\begin{array}{cc}
0 & c(1) \\
e(1) & 0
\end{array}\right)=\left(\begin{array}{cc}
0 & 0.2 \\
0.2 & 0
\end{array}\right), \\
&\left(\begin{array}{cc}
a(2) & 0 \\
0 & b(2)
\end{array}\right)=\left(\begin{array}{cc}
0.3 & 0 \\
0 & 0.2
\end{array}\right), \\
&\left(\begin{array}{cc}
0 & c(2) \\
e(2) & 0
\end{array}\right)=\left(\begin{array}{cc}
0 & 0.2 \\
0.2 & 0
\end{array}\right),
\end{aligned}
$$

and $f(v)=\sin (v), g(u)=(1 / 2)(|u+1|-|u-1|)$, and $K=$ $L=1$. According to Theorem 1 in [9] and Theorem 1 in [29], the neural networks (82) are global exponential stability with $\alpha=1$ and $\beta=1$.

In the presence of stochastic noise and self-feedback matrix $(A, B)^{T}$ uncertainty, the neural networks (82) become

$$
\begin{aligned}
\mathrm{d} u(t, x)= & \left\{D(r(t)) \frac{\partial^{2} u(t, x)}{\partial x^{2}}\right. \\
& -(a(r(t))+\lambda) u(t, x) \\
& +c(r(t)) f(v(t, x))\} \mathrm{d} t \\
& +\sigma u(t, x) \mathrm{d} W(t), \\
\mathrm{d} v(t, x)= & \left\{D^{*}(r(t)) \frac{\partial^{2} v(t, x)}{\partial x^{2}}\right. \\
& -(b(r(t))+\lambda) v(t, x) \\
& +e(r(t)) g(u(t, x))\} \mathrm{d} t \\
+ & \sigma v(t, x) \mathrm{d} W(t) .
\end{aligned}
$$

According to Theorem 6, let $\Delta=0.7>\log (2 \alpha) / \beta=0.6931$ and $\mu_{1}=1$ and $\mu_{2}=2$. From (62), we have

$$
\begin{aligned}
8\left(\sigma^{2}+\right. & \left.0.6 \lambda^{2}\right) \exp \left\{10.08+3.36 \lambda^{2}+5.6 \sigma^{2}\right\} \\
& +2 \exp \{-0.7\}=1 .
\end{aligned}
$$




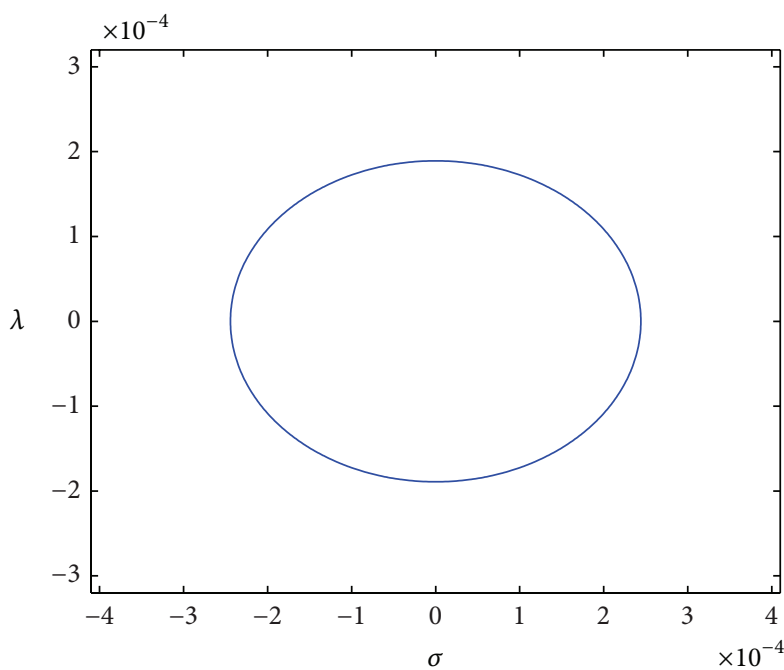

FIGURE 1: The stability region with $(\lambda, \sigma)$ of the neural networks (85).

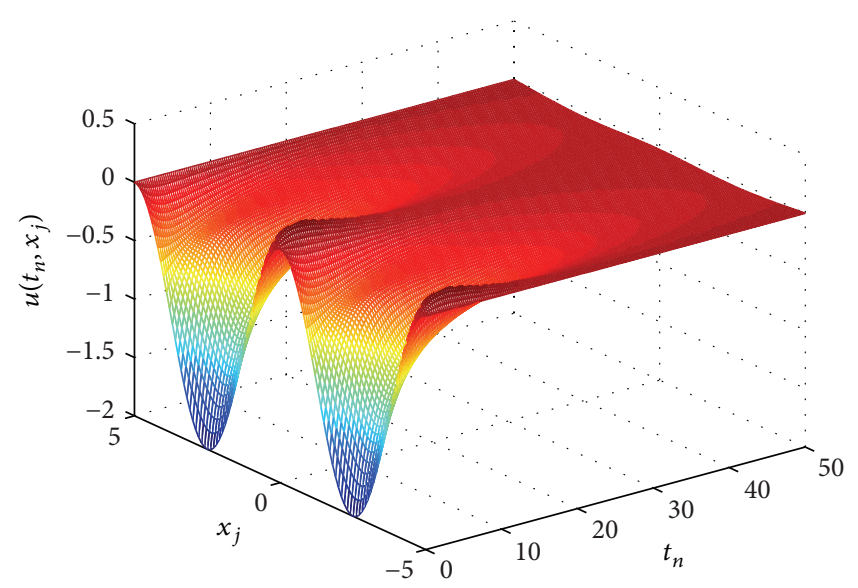

FIGURE 2: Surface curve of $u(t, x)$ of the neural networks (85) in model 1 .

Then, we can obtain its closed curve for $(\lambda, \sigma)$. Figure 1 depicts the stability region for $(\lambda, \sigma)$ in (85).

Figures 2, 3, 4, and 5 depict the surface curves of the neural networks $(85)$ with $(\lambda, \sigma)=\left(-10^{-4}, 10^{-4}\right)$. It shows that the state of the neural networks (85) is mean square globally exponential stability and almost surely globally exponential stable, as the parameter $(\lambda, \sigma)$ in the inner of the curve of Figure 1.

Figures $6,7,8$ and 9 show the surface curves of the neural networks (85) with $(\lambda, \sigma)=(-0.1,1.5)$. It shows that when the conditions in Theorem 6 do not hold, the neural networks (85) become unstable.

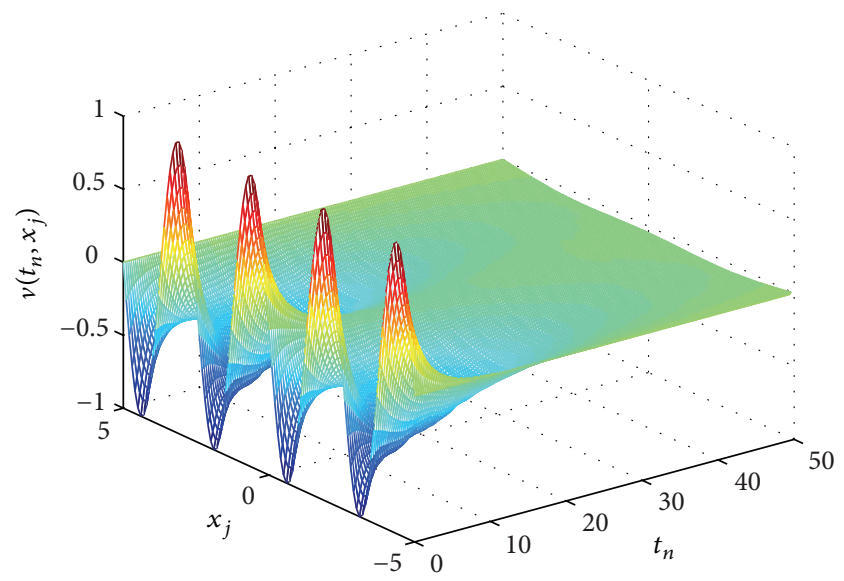

FIgURE 3: Surface curve of $v(t, x)$ of the neural networks (85) in model 1 .

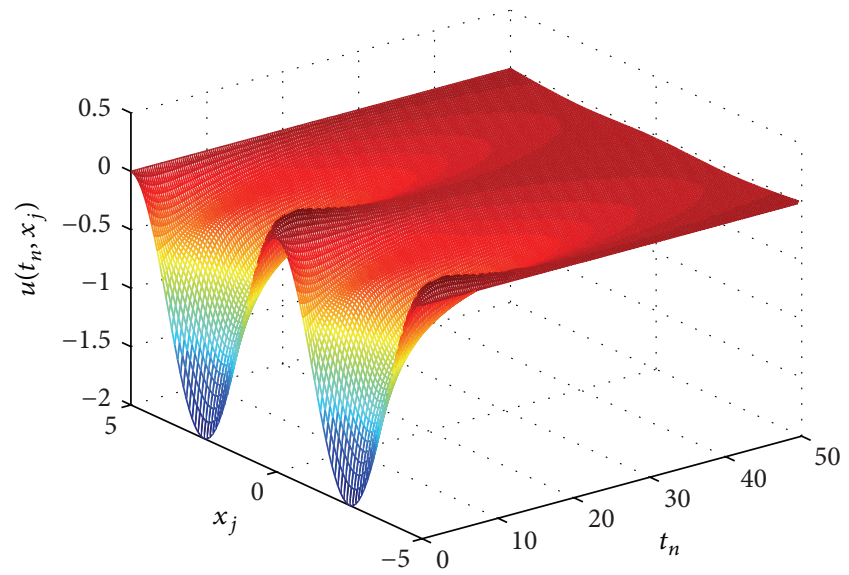

FIGURE 4: Surface curve of $u(t, x)$ of the neural networks (85) in model 2 .

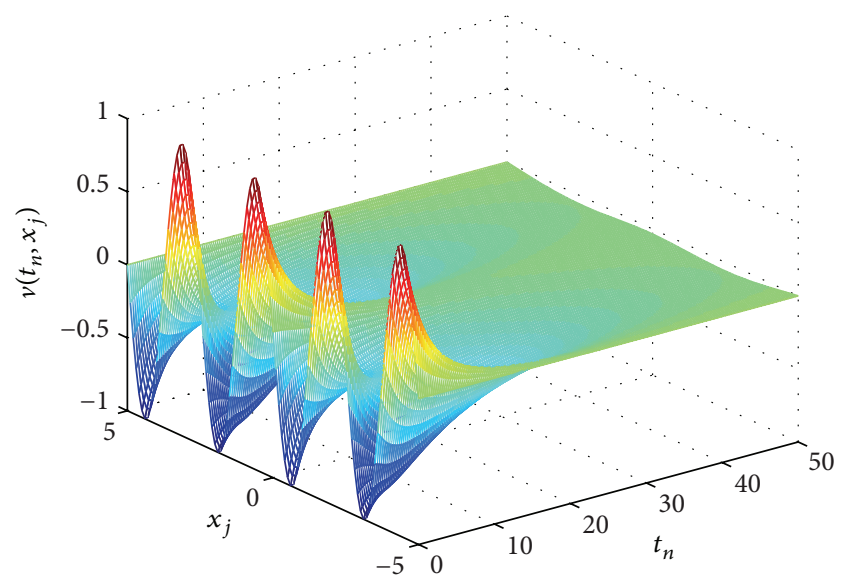

FIGURE 5: Surface curve of $v(t, x)$ of the neural networks (85) in model 2 . 


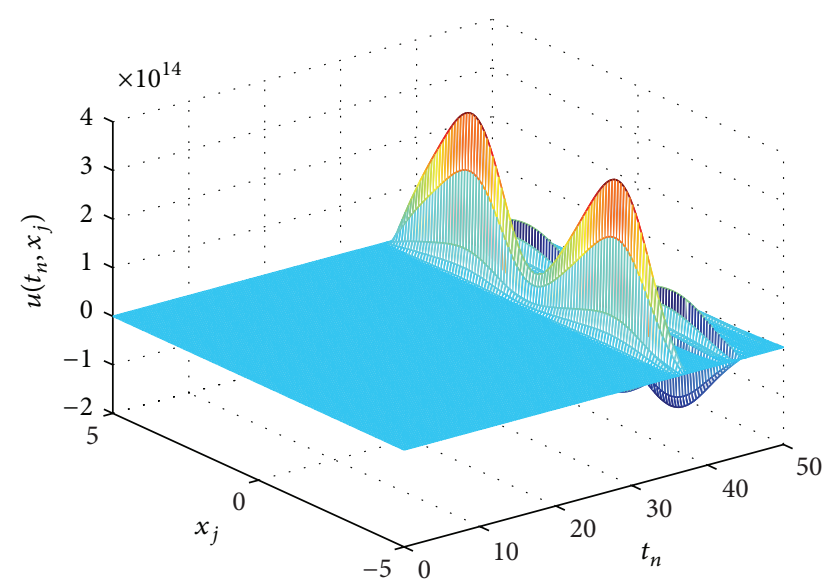

Figure 6: Surface curve of $u(t, x)$ of the neural networks (82) in model 1.

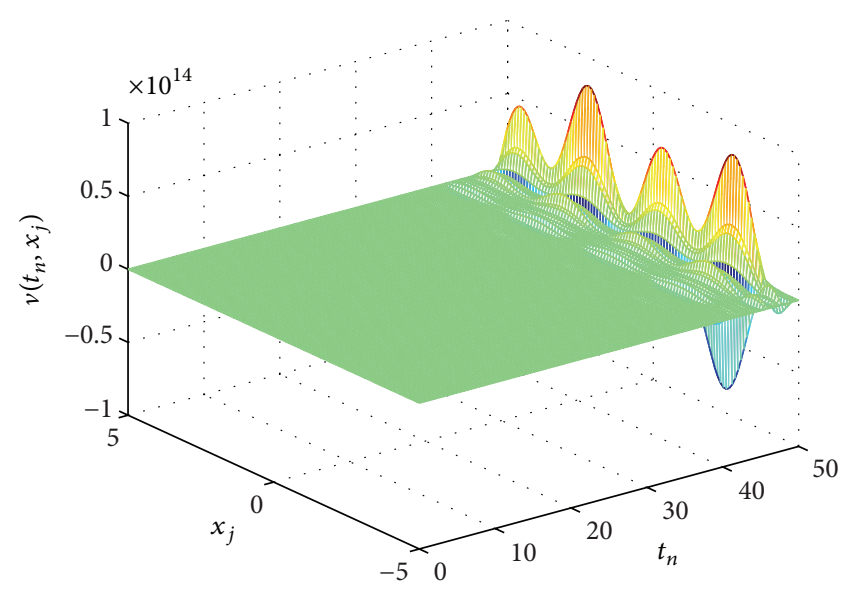

FIgURE 7: Surface curve of $v(t, x)$ of the neural networks (82) in model 1 .

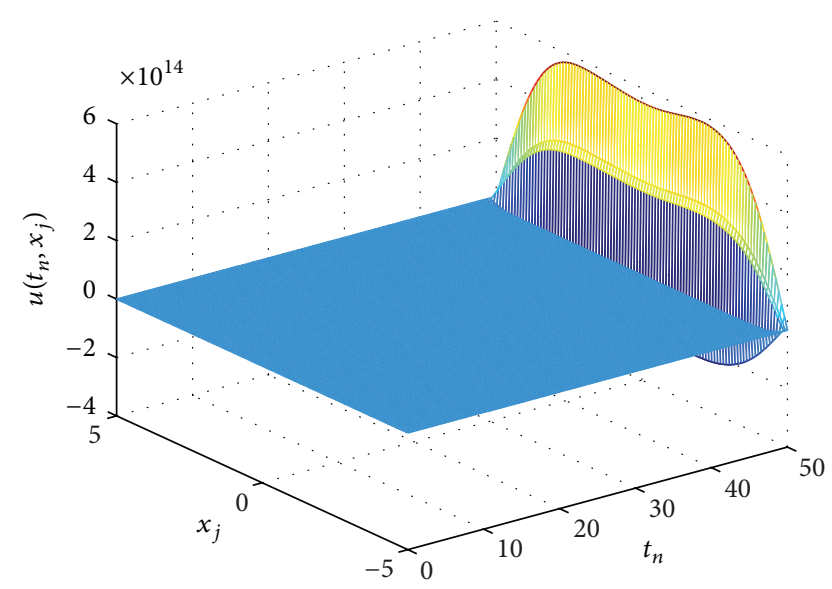

FIGURE 8: Surface curve of $u(t, x)$ of the neural networks (82) in model 2 .

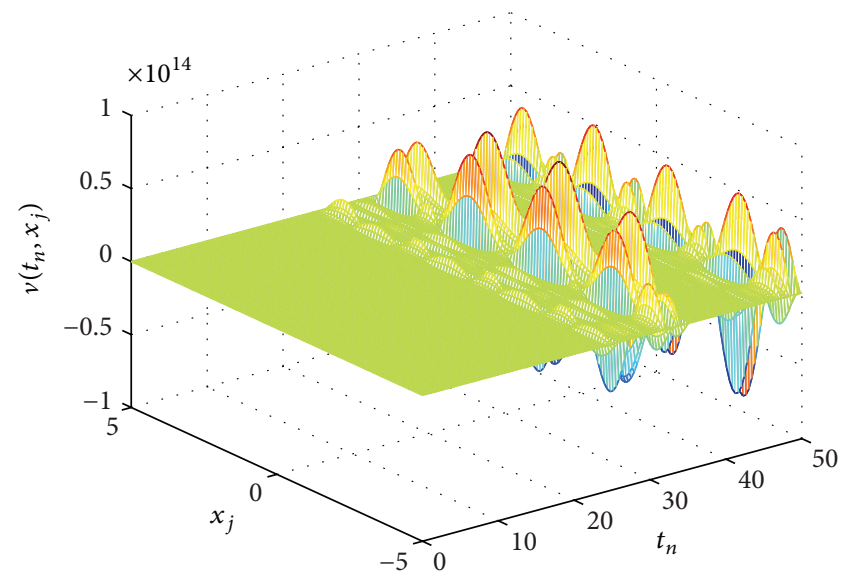

FIGURE 9: Surface curve of $v(t, x)$ of the neural networks (82) in model 2.

\section{Conflict of Interests}

The authors declare that there is no conflict of interests regarding the publication of this paper.

\section{Acknowledgments}

This paper is partially supported by National Natural Science Foundation of China under Grants nos. 61304068, 61374085, and 61134012 and the Fundamental Research Funds for the Central Universities under Grant no. 2013QC019.

\section{References}

[1] B. Kosko, "Adaptive bidirectional associative memories," Applied Optics, vol. 26, no. 23, pp. 4947-4960, 1987.

[2] B. Kosko, "Bidirectional associative memories," IEEE Transactions on Systems, Man, and Cybernetics, vol. 18, no. 1, pp. 49-60, 1988.

[3] B. Kosko, Neural Networks and Fuzzy Systems: A Dynamical Systems Approach to Machine Intelligence, Prentice Hall, Englewood Cliffs, NJ, USA, 1992.

[4] J. Cao and L. Wang, "Exponential stability and periodic oscillatory solution in BAM networks with delays," IEEE Transactions on Neural Networks, vol. 13, no. 2, pp. 457-463, 2002.

[5] A. Chen, J. Cao, and L. Huang, "Exponential stability of BAM neural networks with transmission delays," Neurocomputing, vol. 57, no. 1-4, pp. 435-454, 2004.

[6] P. Balasubramaniam and C. Vidhya, "Global asymptotic stability of stochastic BAM neural networks with distributed delays and reaction-diffusion terms," Journal of Computational and Applied Mathematics, vol. 234, no. 12, pp. 3458-3466, 2010.

[7] B. T. Cui and X. Y. Lou, "Global asymptotic stability of BAM neural networks with distributed delays and reaction-diffusion terms," Chaos, Solitons \& Fractals, vol. 27, no. 5, pp. 1347-1354, 2006.

[8] X. Lou, B. Cui, and W. Wu, "On global exponential stability and existence of periodic solutions for BAM neural networks with distributed delays and reaction-diffusion terms," Chaos, Solitons \& Fractals, vol. 36, no. 4, pp. 1044-1054, 2008. 
[9] Q. Song and J. Cao, "Global exponential stability and existence of periodic solutions in BAM networks with delays and reaction-diffusion terms," Chaos, Solitons \& Fractals, vol. 23, no. 2, pp. 421-430, 2005.

[10] Q. Song, Z. Zhao, and Y. Li, "Global exponential stability of BAM neural networks with distributed delays and reactiondiffusion terms," Physics Letters A, vol. 335, no. 2-3, pp. 213-225, 2005.

[11] Q. Zhu, X. Li, and X. Yang, "Exponential stability for stochastic reaction-diffusion BAM neural networks with time-varying and distributed delays," Applied Mathematics and Computation, vol. 217, no. 13, pp. 6078-6091, 2011.

[12] W. J. Anderson, Continuous-Time Markov Chains: An Applications-Oriented Approach, Springer Series in Statistics: Probability and Its Applications, Springer, New York, NY, USA, 1991.

[13] M. Athans, "Command-and-control (C2) theory: a challenge to control science," IEEE Transactions on Automatic Control, vol. 32, no. 4, pp. 286-293, 1987.

[14] M. Mariton, Jump Linear Systems in Automatic Control, Marcel Dekker, New York, NY, USA, 1990.

[15] H. Huang, D. W. C. Ho, and Y. Qu, "Robust stability of stochastic delayed additive neural networks with Markovian switching," Neural Networks, vol. 20, no. 7, pp. 799-809, 2007.

[16] Y. Shen and J. Wang, "Noise-induced stabilization of the recurrent neural networks with mixed time-varying delays and Markovian-switching parameters," IEEE Transactions on Neural Networks, vol. 18, no. 6, pp. 1857-1862, 2007.

[17] Y. Shen and J. Wang, "Almost sure exponential stability of recurrent neural networks with Markovian switching," IEEE Transactions on Neural Networks, vol. 20, no. 5, pp. 840-855, 2009.

[18] Q. Zhu and J. Cao, "Stability analysis of Markovian jump stochastic BAM neural networks with impulse control and mixed time delays," IEEE Transactions on Neural Networks and Learning Systems, vol. 23, no. 3, pp. 467-479, 2012.

[19] X. X. Liao and X. Mao, "Stability of stochastic neural networks," Neural, Parallel \& Scientific Computations, vol. 4, no. 2, pp. 205224, 1996.

[20] X. X. Liao and X. Mao, "Exponential stability and instability of stochastic neural networks," Stochastic Analysis and Applications, vol. 14, no. 2, pp. 165-185, 1996.

[21] Q. Luo, F. Deng, J. Bao, B. Zhao, and Y. Fu, "Stabilization of stochastic Hopfield neural network with distributed parameters," Science in China. F. Information Sciences, vol. 47, no. 6, pp. 752-762, 2004.

[22] Q. Luo, F. Deng, X. Mao, J. Bao, and Y. Zhang, "Theory and application of stability for stochastic reaction diffusion systems," Science in China. F. Information Sciences, vol. 51, no. 2, pp. 158170, 2008.

[23] X. Mao, "Stability and stabilisation of stochastic differential delay equations," IET Control Theory and Applications, vol. 1, no. 6, pp. 1551-1566, 2007.

[24] H. Zhang, Z. Wang, and D. Liu, "Global asymptotic stability and robust stability of a class of Cohen-Grossberg neural networks with mixed delays," IEEE Transactions on Circuits and Systems. I. Regular Papers, vol. 56, no. 3, pp. 616-629, 2009.

[25] S. Zhu, Y. Shen, and G. Chen, "Exponential passivity of neural networks with time-varying delay and uncertainty," Physics Letters A, vol. 375, no. 2, pp. 136-142, 2010.
[26] K. Liu, Stability of Infinite Dimensional Stochastic Differential Equations with Applications, vol. 135 of Chapman \& Hall/CRC Monographs and Surveys in Pure and Applied Mathematics, Chapman \& Hall/CRC, Boca Raton, Fla, USA, 2006.

[27] X. Mao and C. Yuan, Stochastic Differential Equations with Markovian Switching, Imperial College Press, London, UK, 2006.

[28] D. J. Higham, X. Mao, and C. Yuan, "Preserving exponential mean-square stability in the simulation of hybrid stochastic differential equations," Numerische Mathematik, vol. 108, no. 2, pp. 295-325, 2007.

[29] X. Lou and B. Cui, "Stochastic exponential stability for Markovian jumping BAM neural networks with time-varying delays," IEEE Transactions on Systems, Man, and Cybernetics B: Cybernetics, vol. 37, no. 3, pp. 713-719, 2007. 


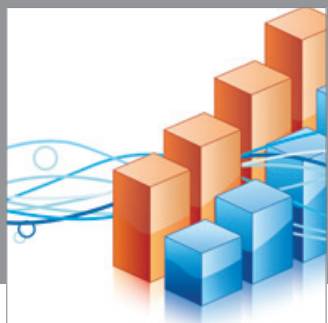

Advances in

Operations Research

mansans

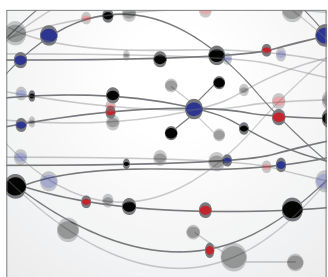

The Scientific World Journal
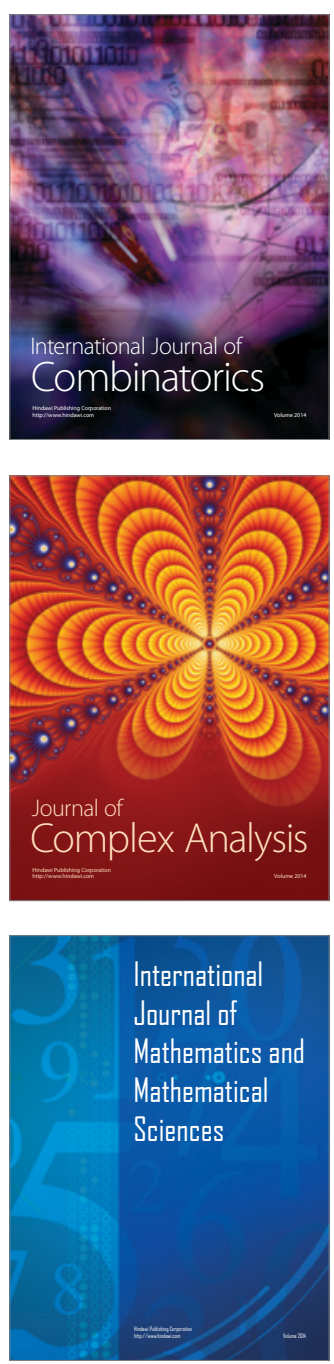
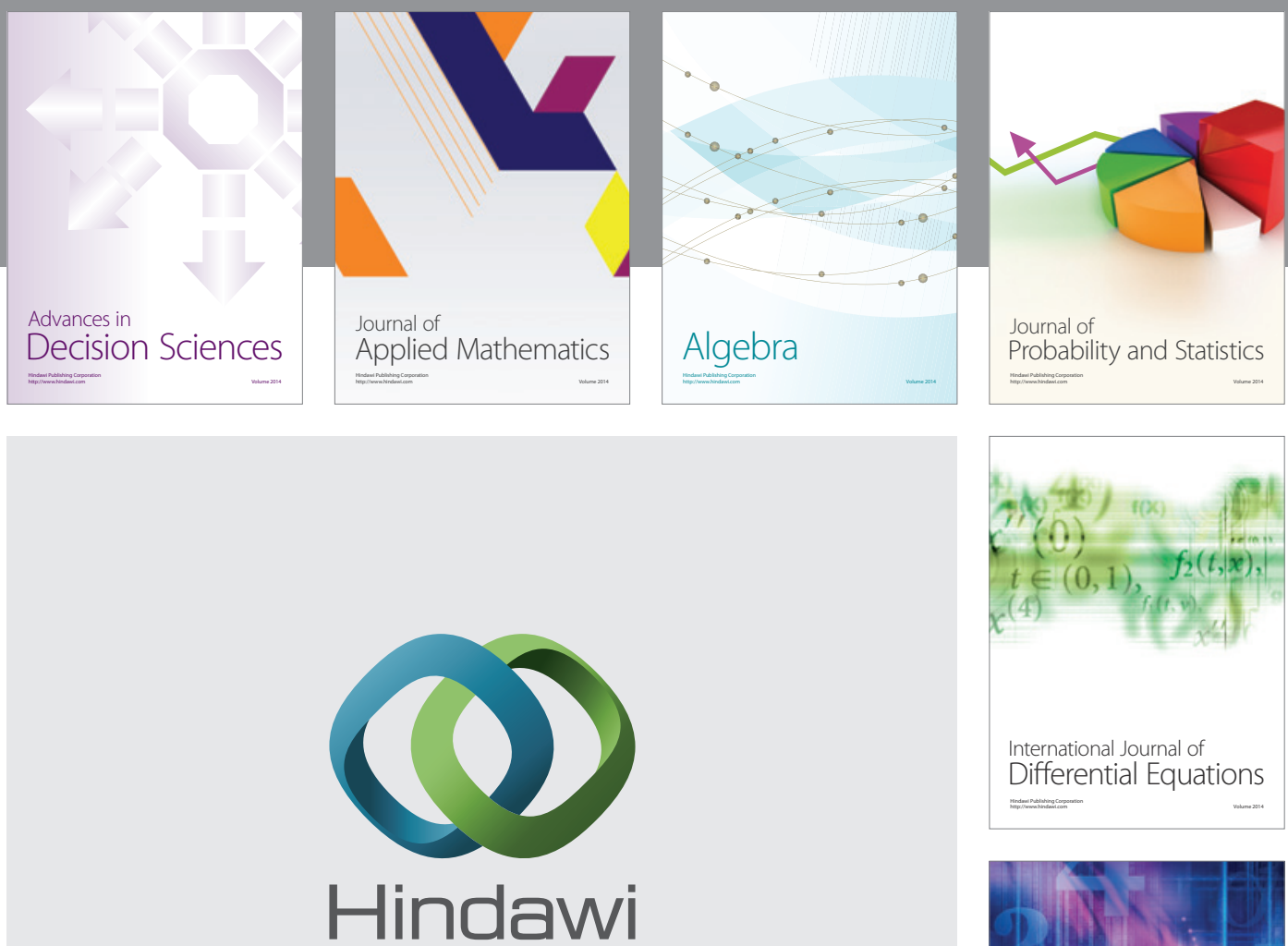

Submit your manuscripts at http://www.hindawi.com
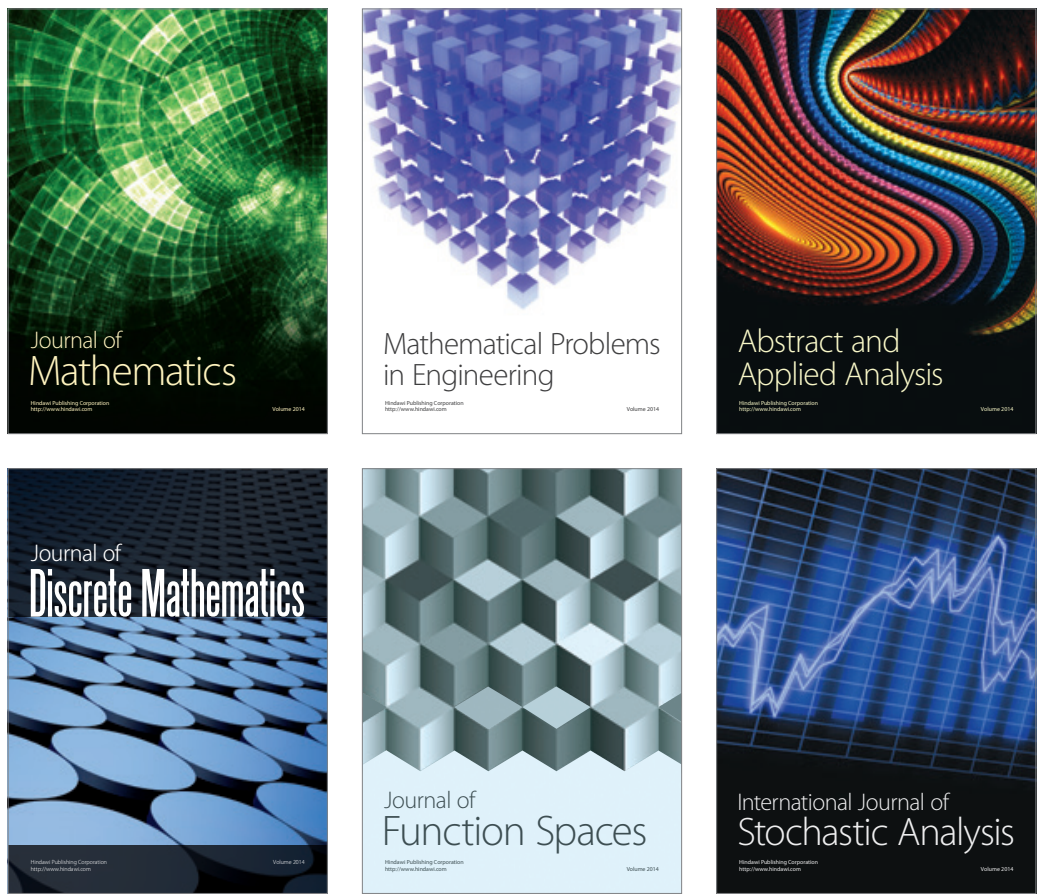

Journal of

Function Spaces

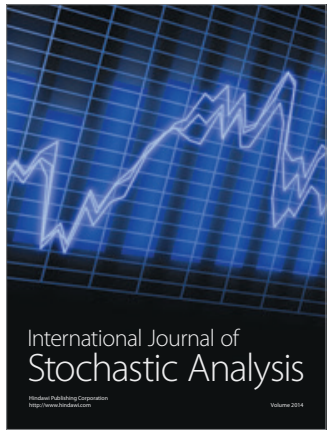

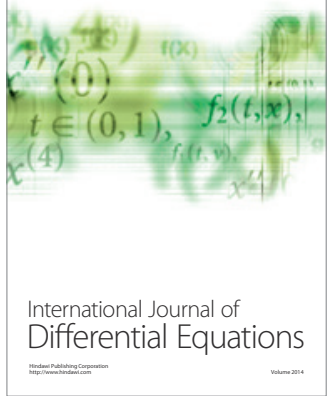
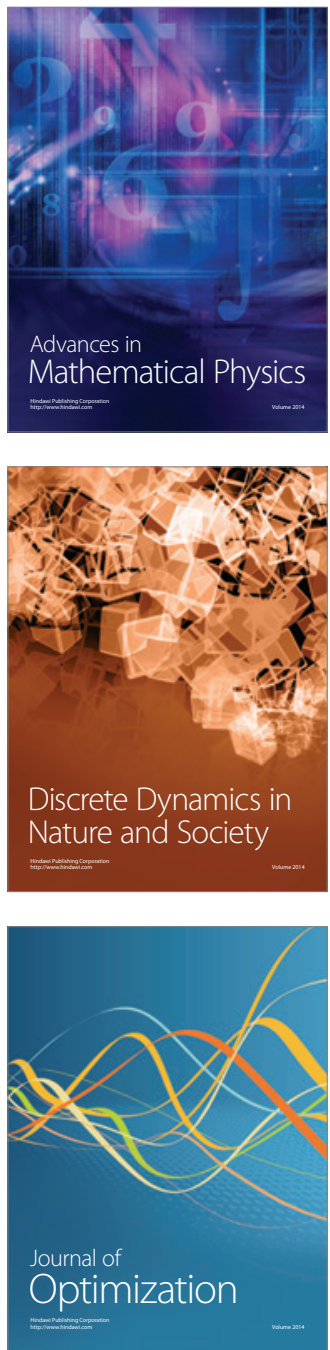